\title{
Predicting the broadband response of a layered cone-cylinder-cone shell
}

\author{
D. Chronopoulos ${ }^{\mathrm{a}, \mathrm{b}}$, M. Ichchou ${ }^{\mathrm{b}}, \mathrm{B}$. Troclet ${ }^{\mathrm{a}, \mathrm{c}}$, O. Bareille ${ }^{\mathrm{b}}$ \\ ${ }^{a}$ EADS Astrium ST, 66 Route de Verneuil, BP3002, 78133 Les Mureaux Cedex, France \\ ${ }^{b}$ Ecole Centrale de Lyon, 36 Avenue Guy de Collongue, 69134 Ecully Cedex, France \\ ${ }^{c}$ Ecole Normale Supérieure de Cachan, 61 Avenue du Président Wilson, 94230 Cachan, France
}

\begin{abstract}
The dynamic response of an aerospace layered structure composed of a combination of conical and cylindrical shells is hereby modelled. In the low and the mid-frequency ranges a WFEM derived ESL approach implemented within a FEM is used in order to predict the response of the shell. Furthermore, in the high frequency range the CLF of the connected subsystems are calculated using a WFEM/FEM approach. These CLF are implemented within a SEA approach in order to predict the structural response. The accuracy and robustness of the developped approaches are exhibited by comparisons to experimental measurements on a layered conical-shell-conical configuration.
\end{abstract}

Keywords: Composite shells, Finite Elements, Statistical Energy Analysis, Experimental validation

\section{Introduction}

Structures made of composite materials and having complex geometric characteristics are extensively used in the modern aerospace industry. More precisely, composite conical-cylindrical combinations of shells are often used as protective payload structures (SYLDA structure of Ariane 5), rocket booster parts, and fuselage components. Modelling the vibroacoustic behaviour of cylindrical and conical shell structures as well as of their combinations is thus essential during the design process of modern aerospace products.

The vibrational modelling of coupled composite conical-cylindrical systems has been an area of sporadic scientific research. Numerical techniques such as the FEM are still considered as the most pertinent approach for modelling coupled composite systems of complex geometries. With regard to the rest of the available models, numerous approaches have been recently published, being roughly divided into modal approaches which aim to predict the free vibration natural frequencies and mode shapes of the system and wave approaches whose aim is the prediction of the wave dispersion and transmission between parts of the system that have homogeneous characteristics. The former type of models is usually used when an accurate description of the global modes of the system is essential for predicting its vibrational behaviour; that is mainly for the low frequency range. On the other hand, modelling the system in a wave context is more suitable for calculating the SEA coupling loss factors between subsystems, which is more suitable for the high frequency range.

Email address: chronopoulos.dimitri@gmail.com (D. Chronopoulos) 
One of the first investigations on the effect of discontinuities on the vibration of thin connected shells is made in [1]. Some years later, in [2] experimental and analytical results were given for a truncated cylinder-cone configuration. In [3] the authors modelled a thin conical-cylindrical shell configuration by implementing the Flügge's equations of motion in a transfer matrix approach. More recently in [4] the problem was solved using a shell FE for which the variational quantities were calculated using the Mindlin theory. In [5] the FE derived results for a thin conicalcylindrical shell combination were verified through experimental measurements. In [6] a power series solution was applied for the calculation of the natural frequencies of segmented axisymmetric shells. Moreover, in [7] the authors analyzed the free vibration of thin coupled shells, also by using a power series method to model the displacement field within the conical part. Both Donnell-Mushtari and Flügge equations of motion were considered. More recently in [8] the free vibration characteristics of a conical-cylindrical section of variable thickness were computed through a Ritz method. However, the structure was assumed to be homogeneous through its thickness.

With regard to modelling the behaviour of coupled shells in a wave-context, the bibliography is not as broad. In [9], the authors included transverse-shear, radial and rotary inertia effects in a bending theory in order to analyze the wave propagation in cylindrical-conical-cylindrical shell configuration. Experimental verification of the results was also provided. In [10] the coupling loss factors of orthotropic curved panels are calculated using a wave dynamic stiffness matrix approach initially introduced in [11].

The WFEM involves the coupling of Periodic Structure Theory (PST) (see [12]) to the FEM. The wave dispersion characteristics within the layered media can be accurately predicted for a very wide frequency range, by solving a polynomial eigenvalue problem for the direction dependant propagation constants. The WFEM for two-dimensional singly curved panels and cylindrical shells has been formulated in [13] by modelling a trapezoid frustum segment of the original curved structure. The wavenumbers and the wave types propagating in the layered shell for each frequency range were computed. The ring frequency of the shell is also correctly predicted.

The main novel points of the work presented in this paper are: 1) The formulation and application of the WFEM derived dynamic stiffness approach presented in $[14,15]$ to a layered conical-cylindrical-conical shell structure. 2) The WFEM derived calculation of the diffused field CLF between coupled two-dimensional layered panels. The CLF are subsequently used within an SEA approach for predicting the response of the layered shell configuration. 3) Experimental measurements on an aerospace conical-cylindrical-conical layered shell are exhibited in order to verify the accuracy and the robustness of the applied approaches.

The paper is organized as follows: In sec.2 the industrial composite SYLDA structure to be tested and modelled is presented. In sec.3 a WFEM derived ESL approach is used in order to predict the dynamic response of the SYLDA structure. The predictions are validated by experimental results. In sec.4 each shell is individually considered as an SEA subsystem. The WFEM is employed in order to calculate the CLF of the shell combination which are used in order to form the SEA coefficients matrix. Conclusions on the presented work are exhibited in sec.5. 


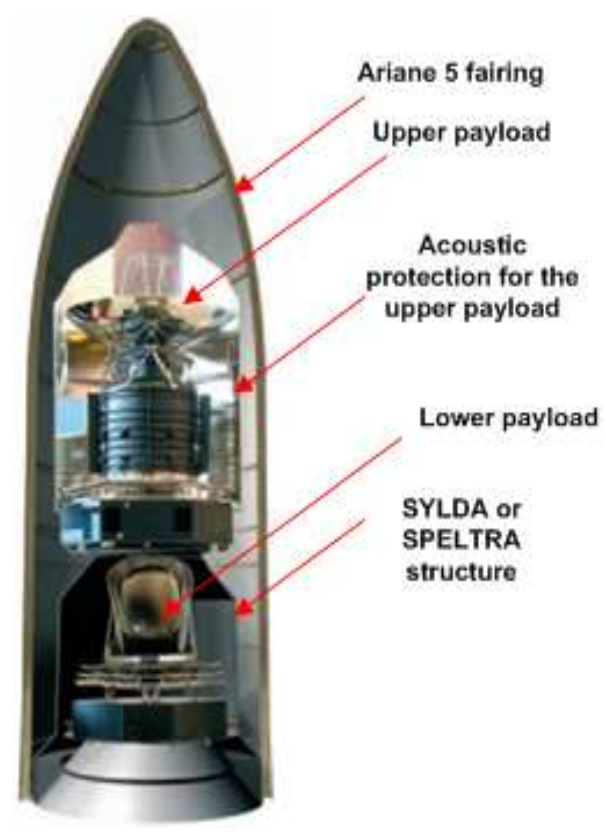

Figure 1: An illustration of the Ariane 5 spacecraft

\section{Presentation of the SYLDA structure}

In this section the industrial composite structure to be experimentally and numerically modelled is exhibited. The configuration to be vibroacoustically analyzed is the SYLDA structure, its name standing for the French acronym of SYstéme de Lancement Double d'Ariane 5. The SYLDA is located inside the launcher fairing (see Fig.1) and allows for multiple payloads to be simultaneously launched.

The SYLDA structure is an assembly of two cones and a cylindrical central part (see Fig.2). The employed materials are of sandwich type with a honeycomb aluminium core and carbon/epoxy made facesheets. Those parts are either bonded together or connected through pyrotechnic cordons which allow the expulsion of the SYLDA parts before placing the payload in orbit. Other connecting elements include springs and Carbon Fibre Reinforced Polymer (CFRP) rings. The structure also includes holes throughout its circumference for giving access to the payload and allowing the atmospheric air to escape during the flight of the vehicle towards the exosphere. All the details regarding the SYLDA structure and its subparts are presented in [16].

In order to conduct an experimental validation on the SYLDA and due to the prohibitive cost of testing an identical to the real SYLDA model, a mock-up of the composite cone-cylinder-cone structure was acquired (see Fig.2). The mock-up structure is a $1 / 4$ scale reproduction of the real one. The exact dimensions of the inner cavity surrounded by the tested structure are shown in Fig.3. All dimensions are in mm. The materials used are of sandwich type; employing $1 \mathrm{~mm}$ thick carbon/epoxy made facesheets (material I) and a $12.7 \mathrm{~mm}$ thick Nomex honeycomb core (material II). The material characteristics are given in Table 1, with coordinate $a$ coinciding with the axial direction of the shell, $c$ with the circumferential direction and $r$ with the radial one. No holes nor stiffeners are included in the mock-up structure. 


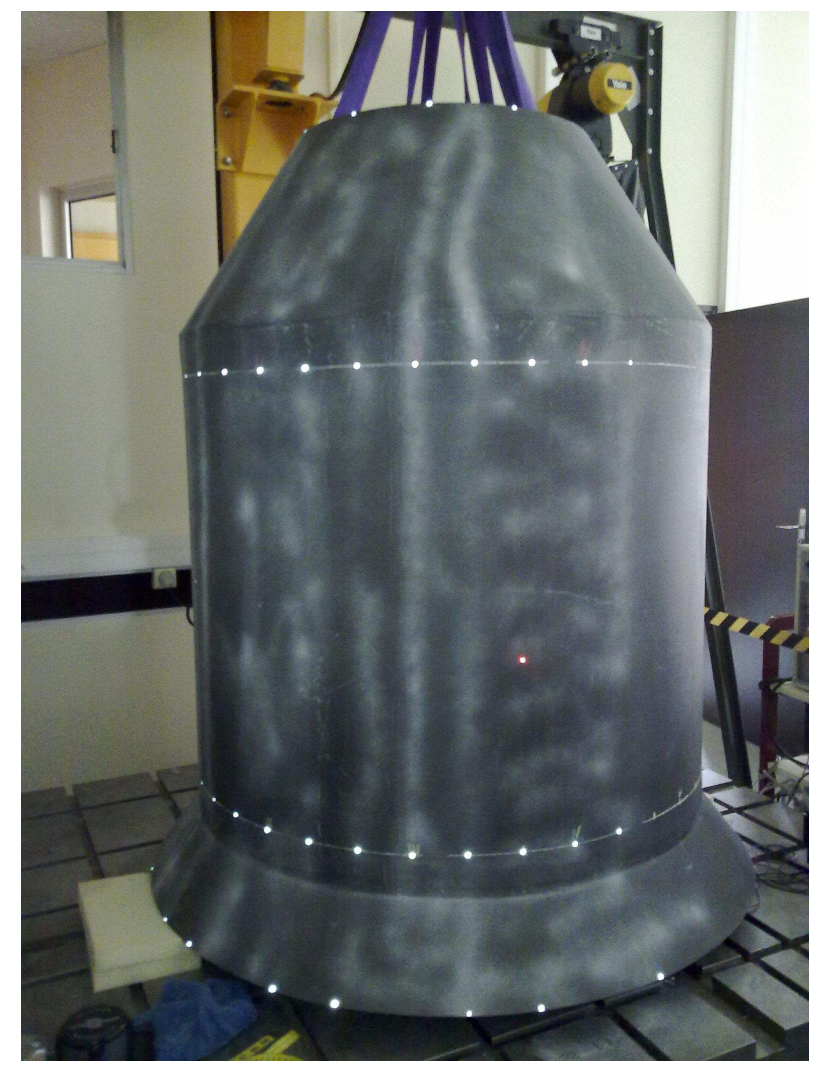

Figure 2: A caption of the SYLDA mock-up used for experimental manipulation

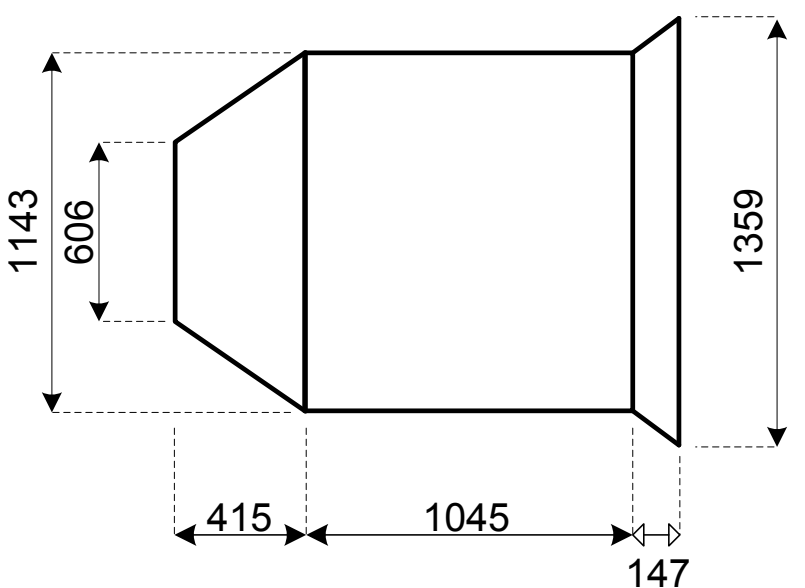

Figure 3: Dimensions of the SYLDA mock-up configuration 


\begin{tabular}{cc}
\multicolumn{2}{c}{ Table 1: Mechanical properties of materials } \\
\hline \hline Material I & Material II \\
\hline$\rho=1530 \mathrm{~kg} / \mathrm{m}^{3}$ & $\rho=63 \mathrm{~kg} / \mathrm{m}^{3}$ \\
$E_{a}=47 \mathrm{GPa}$ & $E_{a}=78 \mathrm{MPa}$ \\
$E_{c}=47 \mathrm{GPa}$ & $E_{c}=78 \mathrm{MPa}$ \\
$v_{a c}=0.1$ & $v_{a c}=0.2$ \\
$G_{a c}=7.4 \mathrm{GPa}$ & - \\
- & $G_{r a}=49 \mathrm{MPa}$ \\
- & $G_{r c}=28 \mathrm{MPa}$ \\
\hline
\end{tabular}

The system comprises the SYLDA structure coupled with its inner and outer acoustic cavities. The structure is freely suspended throughout the experimental testing. The SYLDA is suspended through a metallic cross section which is mounted on a lifting apparatus. The excitor lays inside the shell's cavity and is mounted on the same cross section that supports the shell. In order to avoid a simultaneous rigid motion of the excitor device, a heavy excitor weighting $19 \mathrm{~kg}$ was chosen. Furthermore, in order to avoid slight revolutions of the structure during the testing process, part of the structure's weight was borne by polyurethane made supports to produce friction with the floor. The analysis is separated into two main parts: i) A low to mid-frequency deterministic modelling and ii) a high to mid-frequency SEA modelling approach.

\section{Low to mid-frequency range modelling}

Initially, a full 3D FE model comprising shell elements for the facesheets coupled to solid elements representing the core was developed. The predictions of the 3D FE model are compared to the experimental measurements in order to observe the impact of inevitable parametric uncertainties of the manufactured sandwich shell configuration on its dynamic response. However, this part of the conducted work is not considered as an essential finding, thus it is included as an Appendix. Subsequently, a deterministic numerical modelling of the dynamic response of the SYLDA structure by an ESL approach is attempted. The dynamic stiffness approach presented in [15] is applied to the conical-cylindrical-conical configuration. The peculiarities of the conical substructures are discussed and envisaged by considering the structure to be locally cylindrical. The results of the dynamic stiffness ESL are compared to the full 3D modelling results and the advantages and disadvantages of the approach are commented.

\subsection{Numerical modelling using a dynamic stiffness ESL approach}

In order to apply the ESL approach to a conical-cylindrical shell combination the structure is divided into layers each of which was considered cylindrical for the application of the relations presented in [15] for the dynamic characteristics of the ESL: 


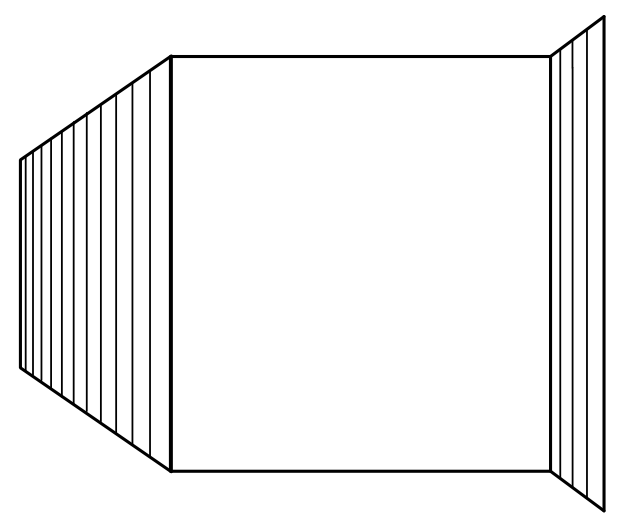

Figure 4: Division of the conical substructures into sections of different material characteristics

$$
\begin{aligned}
& \hat{E}_{a}=\sqrt{\frac{\rho_{s}^{3}}{12 \hat{D}_{a}}} \omega_{r}^{3} R^{3}\left(1-v^{2}\right)=\frac{\rho_{s} \hat{k}_{f, W F E, a}^{2}}{\sqrt{12} \omega} \omega_{r}^{3} R^{3}\left(1-v^{2}\right) \\
& \hat{E}_{c}=\frac{12\left(1-v^{2}\right) \omega^{2} \rho_{s}}{\hat{k}_{f, W F E, c}^{4} \hat{h}^{3}} \\
& \hat{\rho}=\frac{\hat{E}_{a}}{\omega_{r}^{2} R^{2}\left(1-v^{2}\right)}=\frac{\rho_{s} \hat{k}_{f, W F E, a}^{2}}{\sqrt{12} \omega} \omega_{r} R \\
& \hat{h}=\frac{\rho_{s}}{\hat{\rho}}
\end{aligned}
$$

where $f_{r}$ the ring frequency, $\hat{k}_{f, W F E}$ the WFEM calculated flexural wavenumbers of the flat layered panel, $E_{a, c}$ the Young's modulus in the axial and circumferential directions, $v$ the Poisson's ratio, $h$ the thickness of the ESL, $\rho$ its density, $R$ the radius of the shell and`represents the frequency dependence. A mean radius equal to $R=\left(R_{\min }+R_{\max }\right) / 2$ was used for each layer. The number of layers $N$ was selected so that the difference between the ring frequencies of two consecutive layers would be approximately equal to $5 \%$. Better accuracy can effectively be achieved by increasing the number of divisions. The division of the SYLDA structure into layers of different materials and thicknesses is illustrated in Fig.4. The ring frequencies for each of the modelled layers of the composite shell with $N=18$ is shown in Fig.5.

It is straightforward to write the dynamic stiffness matrix of the ESL model by adding the stiffness matrices of each layer as:

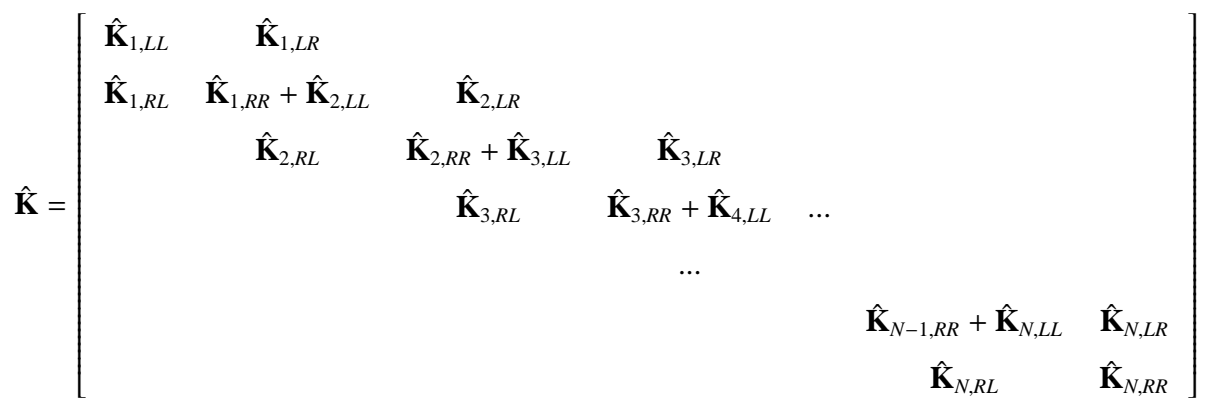

with $L, R$ corresponding to the DoF of the left and the right part of layer respectively. A similar expression applies 


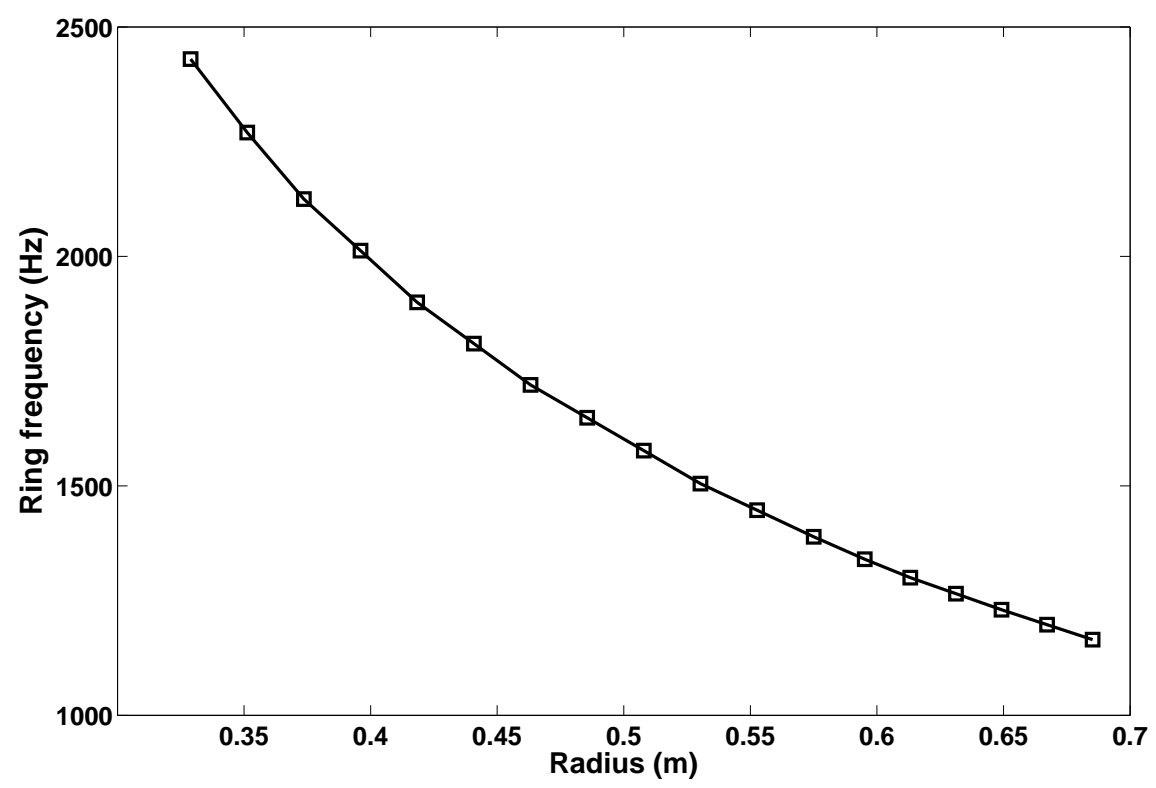

Figure 5: Relation between the radius and the ring frequency of the modelled curved segment

for the mass matrix of the shell.

\subsection{Validation on the SYLDA structure}

The response of the shell is subsequently calculated under an harmonic load. The results are compared to the 3D FE results at an arbitrary point of the structure in Fig.6.

Very good correlation is observed between the ESL and the 3D FE model in the low frequency range (up to $400 \mathrm{~Hz}$ ), with the resonances and the antiresonances being well predicted both in terms of frequency and displacement level. The local modes of the cylindrical shell seem to be well predicted. This fact also suggests that the coupling strength between the subsystems is also well reproduced by the ESL approach. For higher frequencies (above $500 \mathrm{~Hz}$ ) the prediction of the response becomes more sensitive to parametric uncertainties of the layered structure. With regard to the average of the response however, it can be observed that the ESL approach is in very good correlation with the 3D FE model.

In order to compare the predictions concerning the coupling strength between the conical shells and the excited cylindrical substructure, the total (out of plane) vibrational energy of each subsystem is calculated and the results are presented in Figs.7, 8 and 9.

It is observed that the prediction of the energies of the cylindrical and the lower conical subsystems is in very good correlation between the two models. In the low frequency range the peaks and the lows are in excellent correlation to each other both in terms of frequency and energy level. At higher frequencies discrepancies are observed but the average predictions are very well correlated. The coupling strength is therefore very well predicted. With regard to the upper conical shell, the results are in good correlation in the low frequency range, where the local modes of the 


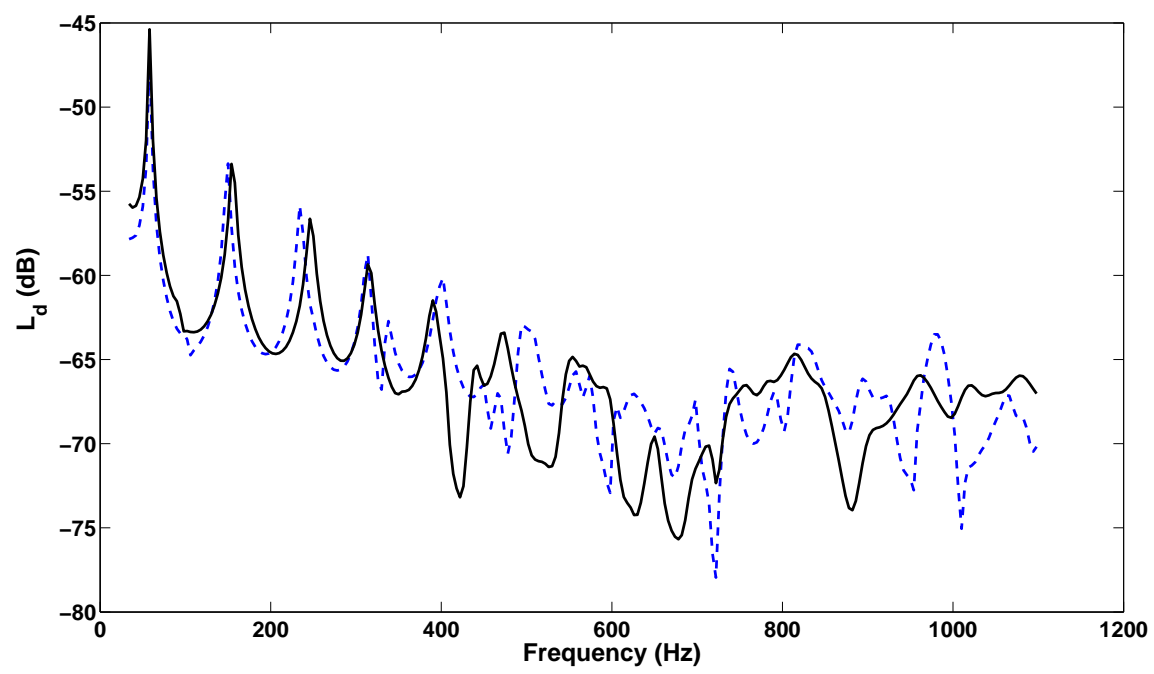

Figure 6: Displacement FRF level comparison at $\left(180^{\circ}, 508 \mathrm{~mm}\right)$ of the cylindrical part: 3D FEM results (-), dynamic stiffness modelling (- -)

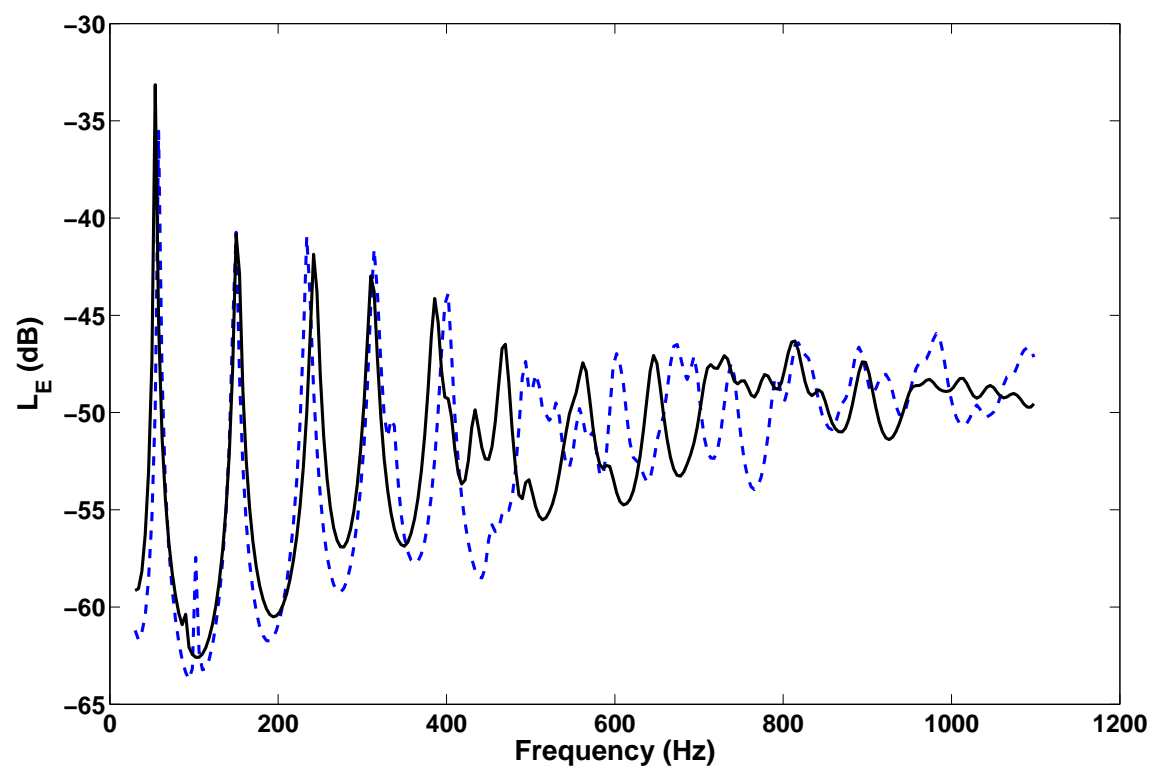

Figure 7: Energy level comparison for the cylindrical part: 3D FEM results (-), dynamic stiffness modelling (- -) 


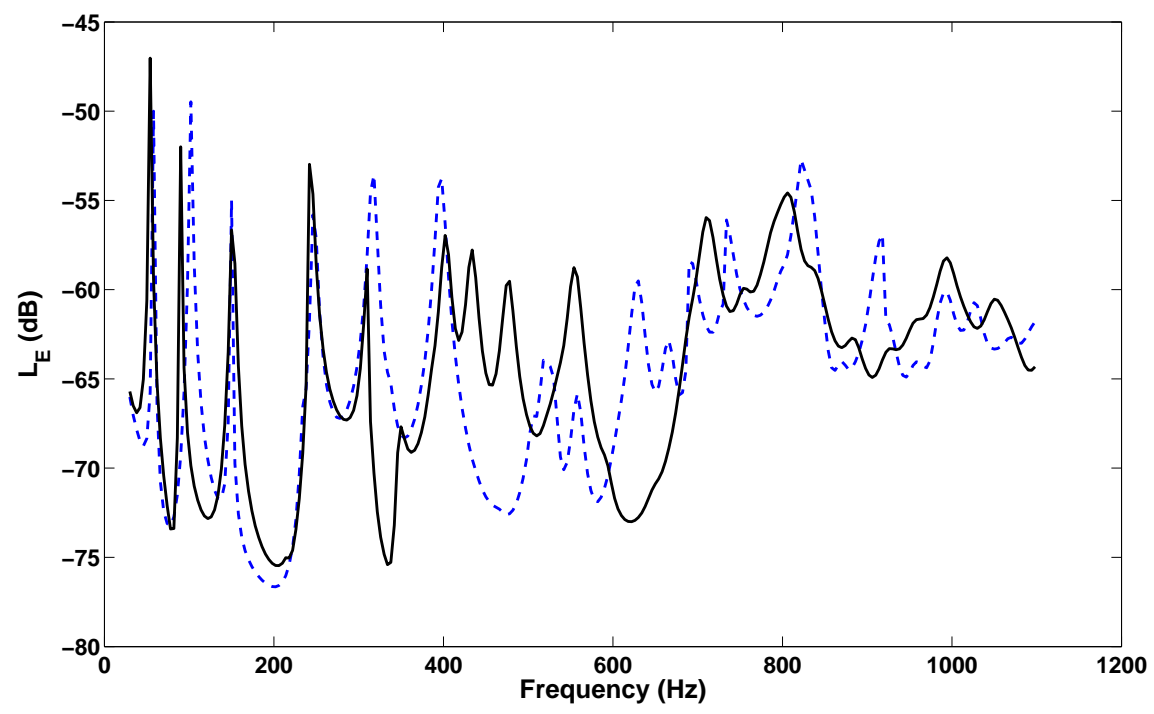

Figure 8: Energy level comparison for the upper conical part: 3D FEM results (-), dynamic stiffness modelling (--)

conical shell are well predicted. Discrepancies between the two models occur between 350 and $500 \mathrm{~Hz}$ probably due to the fact that the coupled shell FE models cannot account for the entirety of the 3D transmission phenomena taking place at the connecting junctions. At higher frequencies -where the behaviour of the upper conical shell evidently becomes more 'local' - the average response predictions are again in good agreement.

\section{SEA modelling of the composite shell assembly}

In the high frequency range where the modal overlap of the structural response of a component is high - mainly due to the increasing modal density of a sandwich structure and the increase of radiation damping - the response can effectively be represented by averaged quadratic quantities. The SEA has been traditionally used for the response prediction in the high frequency domain and is to a large extent based on the accurate calculation of the Coupling Loss Factors (CLF) between the considered subsystems. Hereby, the WFEM will be used in order to revisit the calculation of the CLF between structural layered subsystems. The experimental results obtained on the SYLDA structure will be used to validate the developed models.

\subsection{Coupling Loss Factors calculation for a layered beam assembly}

The considered system comprises two layered structural 1D waveguides (see Fig.10) connected through a connecting element. The approach hereby adopted is presented to a large extent in [17] in order to calculate the transmission and diffusion coefficients for connected 1D waveguides. More recently it was also considered in [18] for the calculation of the transmission efficiency and the response of an ensemble of waveguides. The modelled parts in Fig.10 include the excited waveguide 1 and the receiver waveguide 2 as well as the connecting component. A WFEM/FE analysis is therefore adopted in order to predict the transmission coefficients between the waveguides. 


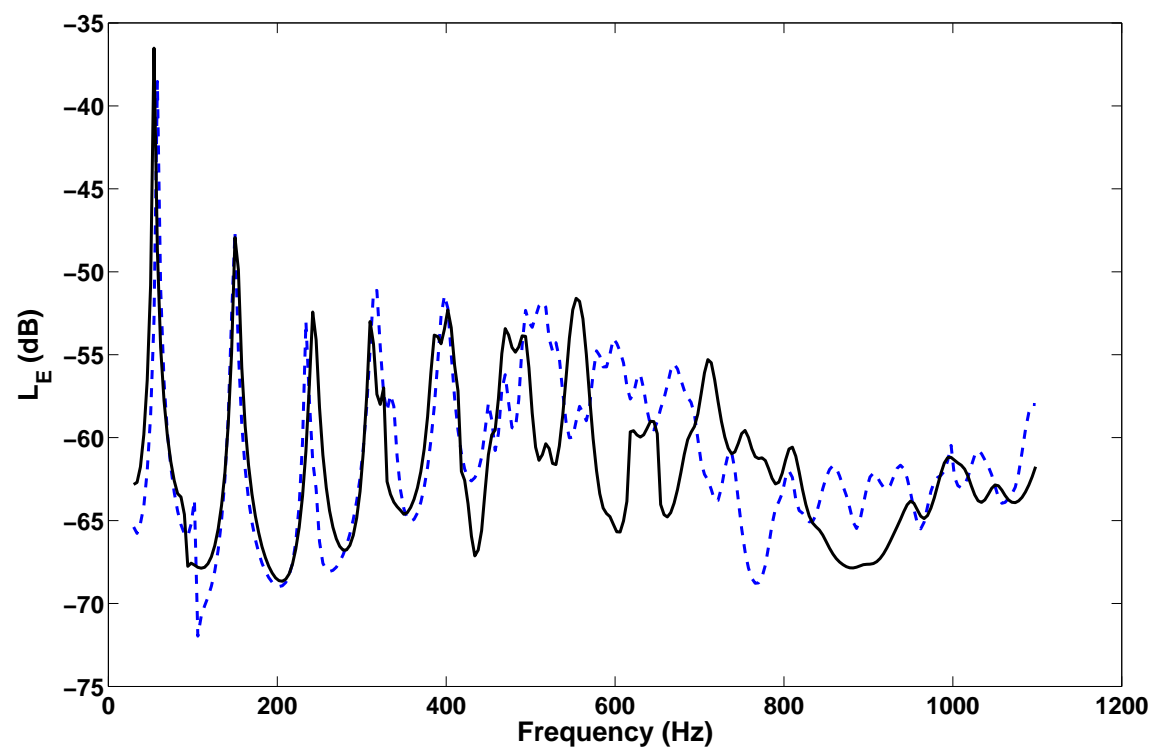

Figure 9: Energy level comparison for the lower conical part: 3D FEM results (-), dynamic stiffness modelling (--)

The wave dispersion characteristics within the semi-infinite waveguides are modelled using the WFEM. The resulting $2 n$ eigenvalues can be associated with $n$ incident waves $\lambda_{i n c, 1 \ldots n}$ and $n$ reflected waves $\lambda_{r e f, n+1 \ldots 2 n}$ with $n$ the number of DoF on each side of the modelled segment and the eigenvalues of the same wave type being related as: $\lambda_{\text {ref }, i}=1 / \lambda_{\text {inc }, i}$. Along with the eigenvalues, the obtained eigenvectors can be classified as:

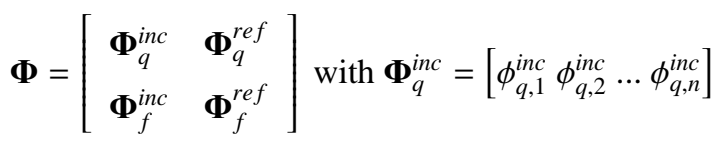

where the subscripts $q, f$ and $1: n$ correspond to the displacement vectors the force vectors and the wave type respectively. Similar expressions hold for $\boldsymbol{\Phi}_{q}^{\text {ref }}, \boldsymbol{\Phi}_{f}^{\text {inc }}$ and $\boldsymbol{\Phi}_{f}^{\text {ref }}$.

The connecting component (surrounded by the dashed line in Fig.10) can be modelled using conventional FE. It is hereby assumed that the DoF of the joint FE model are compatible with the ones of the WFEM models. The harmonic response of the joint segment can be described as:

$$
\left[\begin{array}{lll}
\mathbf{D}_{j_{11}} & \mathbf{D}_{j_{12}} & \mathbf{D}_{j_{1 I}} \\
\mathbf{D}_{j_{21}} & \mathbf{D}_{j_{22}} & \mathbf{D}_{j_{2 l}} \\
\mathbf{D}_{j_{11}} & \mathbf{D}_{j_{I 2}} & \mathbf{D}_{j_{l l}}
\end{array}\right]\left\{\begin{array}{c}
\mathbf{q}_{j}^{1} \\
\mathbf{q}_{j}^{2} \\
\mathbf{q}_{j}^{I}
\end{array}\right\}=\left\{\begin{array}{c}
\mathbf{f}_{j}^{1} \\
\mathbf{f}_{j}^{2} \\
\mathbf{f}_{j}^{I}
\end{array}\right\}
$$

with the subscripts 1,2 and $I$ corresponding to left-side, right-side and internal nodes respectively. Using classical condensation techniques the system of eq.(4) is written as:

$$
\left[\begin{array}{cc}
\mathbf{D}_{j_{11}}-\mathbf{D}_{j_{1 l}} \mathbf{D}_{j_{I I}}^{-1} \mathbf{D}_{j_{l 1}} & \mathbf{D}_{j_{12}}-\mathbf{D}_{j_{1 I}} \mathbf{D}_{j_{I I}}^{-1} \mathbf{D}_{j_{I 2}} \\
\mathbf{D}_{j_{21}}-\mathbf{D}_{j_{2 l}} \mathbf{D}_{j_{I I}}^{-1} \mathbf{D}_{j_{l 1}} & \mathbf{D}_{j_{22}}-\mathbf{D}_{j_{2 l}} \mathbf{D}_{j_{I I}}^{-1} \mathbf{D}_{j_{I 2}}
\end{array}\right]\left\{\begin{array}{c}
\mathbf{q}_{j}^{1} \\
\mathbf{q}_{j}^{2}
\end{array}\right\}=\left\{\begin{array}{c}
\mathbf{f}_{j}^{1} \\
\mathbf{f}_{j}^{2}
\end{array}\right\}
$$




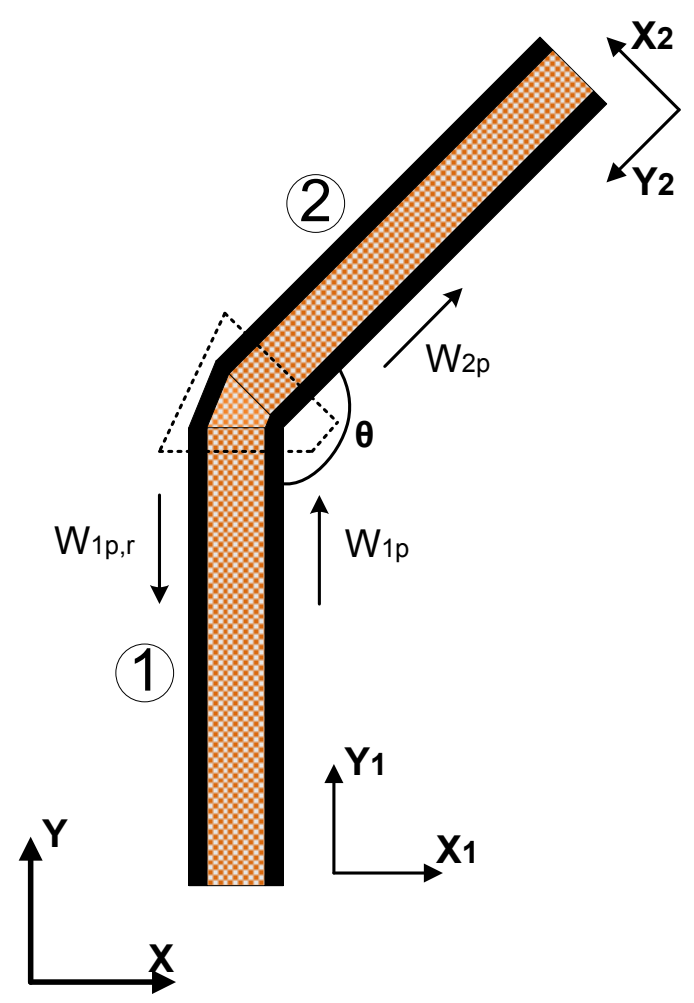

Figure 10: Assembly of two layered 1D waveguides. FE modelled segment surrounded by the dashed line. 
Concerning the structural response of the waveguides, it is shown in [19] that the state vectors $\mathbf{q}_{R}^{1}, \mathbf{f}_{R}^{1}, \mathbf{q}_{L}^{2}, \mathbf{f}_{L}^{2}$ can be written as a superposition function of the wave eigenvectors and the corresponding wave amplitudes $\mathbf{Q}^{\text {inc,i}}, \mathbf{Q}^{\text {ref,i }}$ as:

$$
\begin{gathered}
\left\{\begin{array}{c}
\mathbf{q}_{R}^{1} \\
\mathbf{f}_{R}^{1}
\end{array}\right\}=\left[\begin{array}{ll}
\boldsymbol{\Phi}_{q}^{i n c, 1} & \boldsymbol{\Phi}_{q}^{r e f, 1} \\
\boldsymbol{\Phi}_{f}^{i n c, 1} & \boldsymbol{\Phi}_{f}^{r e f, 1}
\end{array}\right]\left\{\begin{array}{l}
\mathbf{Q}^{i n c, 1} \\
\mathbf{Q}^{r e f, 1}
\end{array}\right\} \\
\left\{\begin{array}{c}
\mathbf{q}_{L}^{2} \\
\mathbf{f}_{L}^{2}
\end{array}\right\}=\left[\begin{array}{ll}
\boldsymbol{\Phi}_{q}^{i n c, 2} & \boldsymbol{\Phi}_{q}^{r e f, 2} \\
\boldsymbol{\Phi}_{f}^{i n c, 2} & \boldsymbol{\Phi}_{f}^{r e f, 2}
\end{array}\right]\left\{\begin{array}{c}
\mathbf{Q}^{i n c, 2} \\
\mathbf{Q}^{r e f, 2}
\end{array}\right\}
\end{gathered}
$$

The continuity conditions at the interfaces of the joint with the waveguides as well as the equilibrium of the system impose that:

$$
\begin{aligned}
& \left\{\begin{array}{c}
\mathbf{q}_{j}^{1} \\
\mathbf{q}_{j}^{2}
\end{array}\right\}=\left[\begin{array}{cc}
\mathbf{R}_{\mathbf{1}} & \mathbf{0} \\
\mathbf{0} & \mathbf{R}_{\mathbf{2}}
\end{array}\right]\left\{\begin{array}{c}
\mathbf{q}_{R}^{1} \\
\mathbf{q}_{L}^{2}
\end{array}\right\} \\
& \left\{\begin{array}{c}
\mathbf{f}_{j}^{1} \\
\mathbf{f}_{j}^{2}
\end{array}\right\}=\left[\begin{array}{cc}
\mathbf{R}_{1} & \mathbf{0} \\
\mathbf{0} & \mathbf{R}_{\mathbf{2}}
\end{array}\right]\left\{\begin{array}{c}
\mathbf{f}_{R}^{1} \\
\mathbf{f}_{L}^{2}
\end{array}\right\}
\end{aligned}
$$

with $\mathbf{R}_{\mathbf{1}}, \mathbf{R}_{\mathbf{2}}$ the transformation matrices for the waveguides 1 and 2 respectively. Using the systems in eq.(5),(6),(7) the matrix containing the transmission and reflection coefficients for each wave type is written as in [17]:

$$
C=-\left[\mathbf{R} \Phi_{f}^{r e f}-\mathbf{D}_{j} \mathbf{R} \Phi_{q}^{r e f}\right]^{-1}\left[\mathbf{R} \Phi_{f}^{i n c}-\mathbf{D}_{j} \mathbf{R} \Phi_{q}^{i n c}\right]
$$

$$
\begin{gathered}
\text { with } \mathbf{R}=\left[\begin{array}{cc}
\mathbf{R}_{\mathbf{1}} & \mathbf{0} \\
\mathbf{0} & \mathbf{R}_{\mathbf{2}}
\end{array}\right], \boldsymbol{\Phi}_{f}^{r e f}=\left[\begin{array}{cc}
\boldsymbol{\Phi}_{f}^{r e f, 1} & \mathbf{0} \\
\mathbf{0} & \boldsymbol{\Phi}_{f}^{r e f, 2}
\end{array}\right], \boldsymbol{\Phi}_{q}^{r e f}=\left[\begin{array}{cc}
\boldsymbol{\Phi}_{q}^{r e f, 1} & \mathbf{0} \\
\mathbf{0} & \boldsymbol{\Phi}_{q}^{r e f, 2}
\end{array}\right], \boldsymbol{\Phi}_{f}^{i n c}=\left[\begin{array}{cc}
\boldsymbol{\Phi}_{f}^{i n c, 1} & \mathbf{0} \\
\mathbf{0} & \boldsymbol{\Phi}_{f}^{i n c, 2}
\end{array}\right], \boldsymbol{\Phi}_{q}^{i n c}= \\
{\left[\begin{array}{cc}
\boldsymbol{\Phi}_{q}^{i n c, 1} & \mathbf{0} \\
\mathbf{0} & \boldsymbol{\Phi}_{q}^{i n c, 2}
\end{array}\right] \text { and }} \\
\mathbf{D}_{j}=\left[\begin{array}{ll}
\mathbf{D}_{j_{11}}-\mathbf{D}_{j_{1 I}} \mathbf{D}_{j_{I I}}^{-1} \mathbf{D}_{j_{I I}} & \mathbf{D}_{j_{12}}-\mathbf{D}_{j_{1 I}} \mathbf{D}_{j_{I I}}^{-1} \mathbf{D}_{j_{I 2}} \\
\mathbf{D}_{j_{21}}-\mathbf{D}_{j_{2 l}} \mathbf{D}_{j_{I I}}^{-1} \mathbf{D}_{j_{I 1}} & \mathbf{D}_{j_{22}}-\mathbf{D}_{j_{2 l}} \mathbf{D}_{j_{I I}}^{-1} \mathbf{D}_{j_{I 2}}
\end{array}\right] .
\end{gathered}
$$

The CLF between the wave type $p$ in the waveguide 1 and the wave type $z$ propagating within the waveguide 2 can be computed using the classical relation:

$$
\eta_{p_{1}, z_{2}}=\frac{\tau_{p_{1}, z_{2}} c_{g, p_{1}}}{2 \omega L_{1}}
$$

with $L_{1}$ the length of waveguide $1, c_{g, p_{1}}$ the group velocity of the incident wave and $\tau_{p_{1}, z_{2}}$ the transmission efficiency between the two wave types expressed as:

$$
\tau_{p_{1}, z_{2}}=\frac{P_{T, z_{2}}}{P_{I, p_{1}}}
$$


with $P_{T, z_{2}}$ the transmitted power in waveguide 2 and $P_{I, p_{1}}$ the incident power in waveguide 1 . Using the expressions presented in [20] the energy densities for a waveguide within which a wave $z$ is propagating are written as:

$$
\begin{gathered}
E_{K, z}^{i n c}=\frac{\omega^{2}}{4 L_{s}} \operatorname{Re}\left(\left\{\begin{array}{c}
\boldsymbol{\Phi}_{q, z}^{i n c} \\
\lambda_{z}^{i n c} \boldsymbol{\Phi}_{q, z}^{i n c}
\end{array}\right\}^{H} \mathbf{M}_{s}\left\{\begin{array}{c}
\boldsymbol{\Phi}_{q, z}^{i n c} \\
\lambda_{z}^{i n c} \boldsymbol{\Phi}_{q, z}^{i n c}
\end{array}\right\}\right) \\
E_{P, z}^{i n c}=\frac{1}{4 L_{s}} \operatorname{Re}\left(\left\{\begin{array}{c}
\boldsymbol{\Phi}_{q, z}^{i n c} \\
\lambda_{z}^{i n c} \boldsymbol{\Phi}_{q, z}^{i n c}
\end{array}\right\}^{H} \mathbf{K}_{s}\left\{\begin{array}{c}
\boldsymbol{\Phi}_{q, z}^{i n c} \\
\lambda_{z}^{i n c} \boldsymbol{\Phi}_{q, z}^{i n c}
\end{array}\right\}\right) \\
E_{T, z}^{i n c}=E_{K, z}^{i n c}+E_{P, z}^{i n c}
\end{gathered}
$$

with $E_{K, z}, E_{P, z}, E_{T, z}$ the kinetic, potential and total energy densities and $\mathbf{M}_{s}, \mathbf{K}_{s}$ the mass and stiffness matrices of the modelled segment of the waveguide. The power due to the passage of a wave type $z$ propagating within a waveguide can be written as:

$$
P_{I, z}^{i n c}=\frac{1}{2} \operatorname{Re}\left(i \omega\left(Q_{z}^{i n c}\right)^{H}\left\{\boldsymbol{\Phi}_{f, z}^{i n c}\right\}^{H} \boldsymbol{\Phi}_{q, z}^{i n c} Q_{z}^{i n c}\right)
$$

The transmission efficiency between the two waves can therefore be written as:

$$
\tau_{p_{1}, z_{2}}=\frac{\operatorname{Re}\left(i \omega\left(Q_{z_{2}}^{r e f}\right)^{H}\left\{\boldsymbol{\Phi}_{f, z_{2}}^{r e f}\right\}^{H} \boldsymbol{\Phi}_{q, z_{2}}^{r e f} Q_{z_{2}}^{r e f}\right)}{\operatorname{Re}\left(i \omega\left(Q_{p_{1}}^{i n c}\right)^{H}\left\{\boldsymbol{\Phi}_{f, p_{1}}^{i c}\right\}^{H} \boldsymbol{\Phi}_{q, p_{1}}^{i n c} Q_{p_{1}}^{i n c}\right)}
$$

However the ratio of the amplitudes of the two waves can be found in matrix $C$, therefore eq.(13) becomes similar to the expression given in [21]:

$$
\tau_{p_{1}, z_{2}}=\frac{\operatorname{Re}\left(i \omega\left\{\boldsymbol{\Phi}_{f, z_{2}}^{r e f}\right\}^{H} \boldsymbol{\Phi}_{q, z_{2}}^{r e f}\right)\left|C_{p_{1}, z_{2}}\right|^{2}}{\operatorname{Re}\left(i \omega\left\{\boldsymbol{\Phi}_{f, p_{1}}^{i n c}\right\}^{H} \boldsymbol{\Phi}_{q, p_{1}}^{i n c}\right)}
$$

The group velocity of the wavetype is written as in [20]:

$$
c_{g, z}^{i n c}=\frac{P_{I, z}^{i n c}}{E_{T, z}^{i n c}}
$$

Therefore by introducing eq.(14),(15) into (9) it is straightforward to calculate the CLF between two waves propagating in the layered $1 \mathrm{D}$ waveguides.

\subsection{Coupling Loss Factors (CLF) calculation for coupled layered 2D panels}

Two layered panels are assumed to be connected through a joint (see Fig.11). The diffused field CLF between the two panels are to be calculated. Compared to the methodology described in sec.4.1 the analysis now differs. 


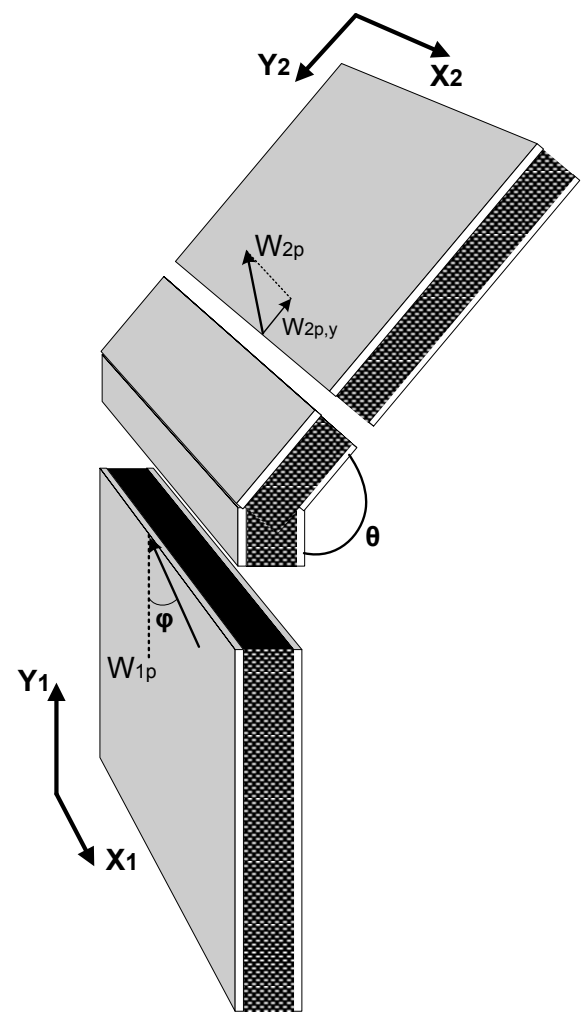

Figure 11: Assembly of two layered plates in an angle; descritized into the excited panel (subsystem 1), the 2D joint and the inclined panel (subsystem 2).

A 2D WFE analysis is initially conducted for the two panels. The wavenumbers propagating in parallel to the joint (say $k_{x}$ ) as well as the frequency are fixed for the nonlinear eigenproblem and the corresponding solutions for each set of $k_{x}, f$ are sought. The wavenumber maps for the two connected panels can be plotted as a function of frequency and direction of propagation. By interpolating on these maps the wavenumber $k$ for every angle of incidence $\phi$ is therefore known.

The diffused field CLF for two anisotropic panels is taken as in [10]:

$$
\eta_{p_{1}, z_{2}}=\frac{L_{12}}{2 \pi^{2} n_{p_{1}}} \int_{\phi_{\min }}^{\phi_{\max }} \frac{c_{g x, p_{1}} \tau_{p_{1}, z_{2}}}{c_{p_{1}} c_{g, p_{1}}} d \phi
$$

with $L_{12}, n_{p_{1}}$ the connection length of the two panels and the modal density of $p$ wave type in panel $1, c_{p_{1}}, c_{g, p_{1}}$ the phase and group velocities of $p$ wave type in panel 1 and $\tau_{p_{1}, z_{2}}$ the angle dependent transmission efficiency of $p$ wave type in panel 1 to the $z$ wave type in panel 2 . Once the wavenumbers $k_{x}, k_{y}$ for every $\phi$ are known the problem can be considered as a one dimensional problem described in sec.4.1, with the wave mode shapes in waveguide 1 now equal to: 


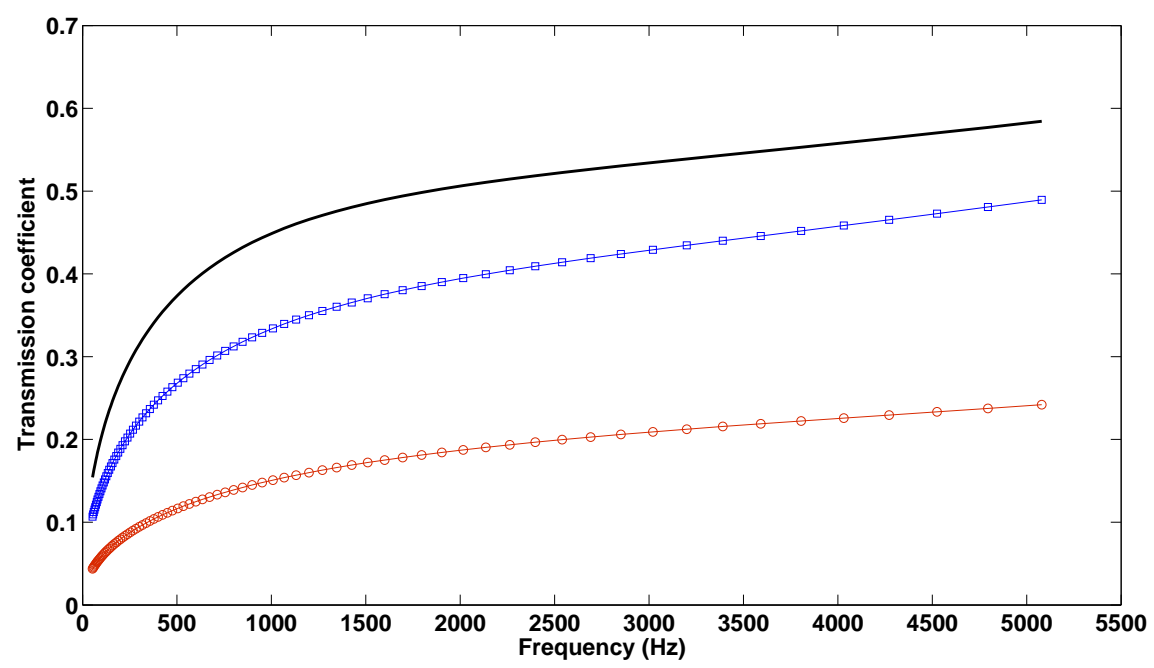

Figure 12: Transmission coefficients for a flexural/flexural coupling of the layered panels with $\theta=32,9^{\circ}$ and $\phi=0(-), \phi=30(\square), \phi=60(o)$

$$
\boldsymbol{\Phi}_{q, f}^{i n c, 1}=\mathbf{R}_{\phi} \boldsymbol{\Phi}_{q, f}^{i n c, 1,2 D}
$$

With $\boldsymbol{\Phi}_{q, f}^{i n c, 1,2 D}$ the wave mode shapes as predicted by the 2D WFEM and $\mathbf{R}_{\phi}$ a transformation matrix applied to account for the angle of incidence $\phi$. The transmission and reflection coefficients can therefore be computed as before:

$$
\mathcal{C}(\phi)=-\left[\mathbf{R} \boldsymbol{\Phi}_{f}^{r e f}-\mathbf{D}_{j} \mathbf{R} \boldsymbol{\Phi}_{q}^{r e f}\right]^{-1}\left[\mathbf{R} \boldsymbol{\Phi}_{f}^{i n c}-\mathbf{D}_{j} \mathbf{R} \boldsymbol{\Phi}_{q}^{i n c}\right]
$$

this time with $\mathbf{R}=\left[\begin{array}{cc}\mathbf{R}_{\mathbf{1}, \theta} \mathbf{R}_{\mathbf{1}, \phi} & \mathbf{0} \\ \mathbf{0} & \mathbf{R}_{\mathbf{2}, \theta}\end{array}\right]$.

The $\phi$ dependent transmission efficiency can therefore be calculated and by introducing it into eq.(16) the diffused field CLF can be predicted. Is is reminded that anisotropic panels may actually carry energy away from the connecting interface. Therefore the integration in eq.(16) should be conducted only for angles for which the group velocity perpendicular to the junction $c_{g x, p_{1}}$ quantity is positive.

Numerical examples of the described approach are then exhibited. The approach is applied to two flat layered panels coupled with an angle $\theta=32,9^{\circ}$ (corresponding to the connecting angle of the upper conical shell) and having the same characteristics as the sandwich material of the SYLDA model, with directions $x$ and $y$ coinciding with directions $c$ and $a$ respectively. The transmission coefficients for a flexural/flexural wave coupling are shown in Fig.12.

The results are qualitatively in agreement with analytical solutions for thin isotropic structures (see [22]) which suggest that the transmission coefficient decreases when increasing the angle of attack $\phi$. Analytical solutions for coupled layered thick structures are particularly difficult, if not impossible to be encountered. The reflection coefficients for the same type of coupling are exhibited in Fig.13. The same comments apply to the reflection coefficients for 


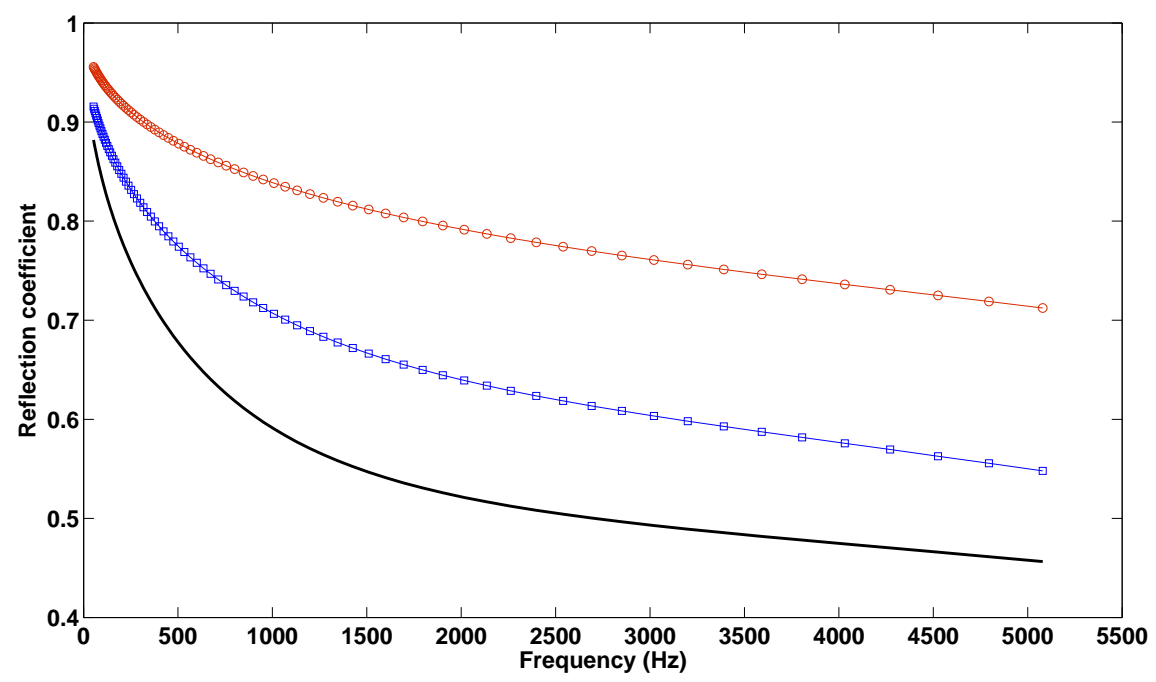

Figure 13: Reflection coefficients for a flexural/flexural coupling of the layered panels with $\theta=32,9^{\circ}$ and $\phi=0(-), \phi=30$ ( $\square$ ), $\phi=60$ (o)

being qualitatively in correlation with analytical results.

\subsection{SEA analysis of the SYLDA}

An SEA analysis of the SYLDA structure is hereby conducted in order to compute the structural response of each substructure. The three layered shells are considered as separate subsystems. A qualitative presentation of the power exchange between the subsystems is given in Fig.14.

The SEA equations can be formulated as:

$$
\left[\begin{array}{ccc}
\eta_{d, 1}+\eta_{12}+\eta_{\text {rad }, 1} & -\eta_{21} & 0 \\
-\eta_{12} & \eta_{d, 2}+\eta_{21}+\eta_{23}+\eta_{r a d, 2} & -\eta_{32} \\
0 & -\eta_{23} & \eta_{d, 3}+\eta_{32}+\eta_{r a d, 3}
\end{array}\right]\left\{\begin{array}{c}
E_{1} \\
E_{2} \\
E_{3}
\end{array}\right\}=\left\{\begin{array}{c}
0 \\
\frac{W_{2, i n j}}{\omega} \\
0
\end{array}\right\}
$$

with $E_{i}$ the spatially averaged total energy of subsystem $i, W_{2, i n j}$ the power injected in subsystem $2, \eta_{\text {rad }, i}$ the Radiation Loss Factor (RLF) of subsystem $i$ and $\eta_{d, i}$ the Dissipation Loss Factor (DLF) of subsystem $i$. Writing the solution of the system as:

$$
\left\{\begin{array}{c}
\frac{\omega E_{1}}{W_{2, i n j}} \\
\frac{\omega E_{2}}{W_{2, i n j}} \\
\frac{\omega E_{3}}{W_{2, i n j}}
\end{array}\right\}=\mathbf{A}^{-1}\left\{\begin{array}{l}
0 \\
1 \\
0
\end{array}\right\}
$$

$$
\text { with } \mathbf{A}=\left[\begin{array}{ccc}
\eta_{d, 1}+\eta_{12}+\eta_{\text {rad }, 1} & -\eta_{21} & 0 \\
-\eta_{12} & \eta_{d, 2}+\eta_{21}+\eta_{23}+\eta_{\text {rad }, 2} & -\eta_{32} \\
0 & -\eta_{23} & \eta_{d, 3}+\eta_{32}+\eta_{\text {rad }, 3}
\end{array}\right] \text {, it is now straightforward that the }
$$

energy ratios will be equal to: 


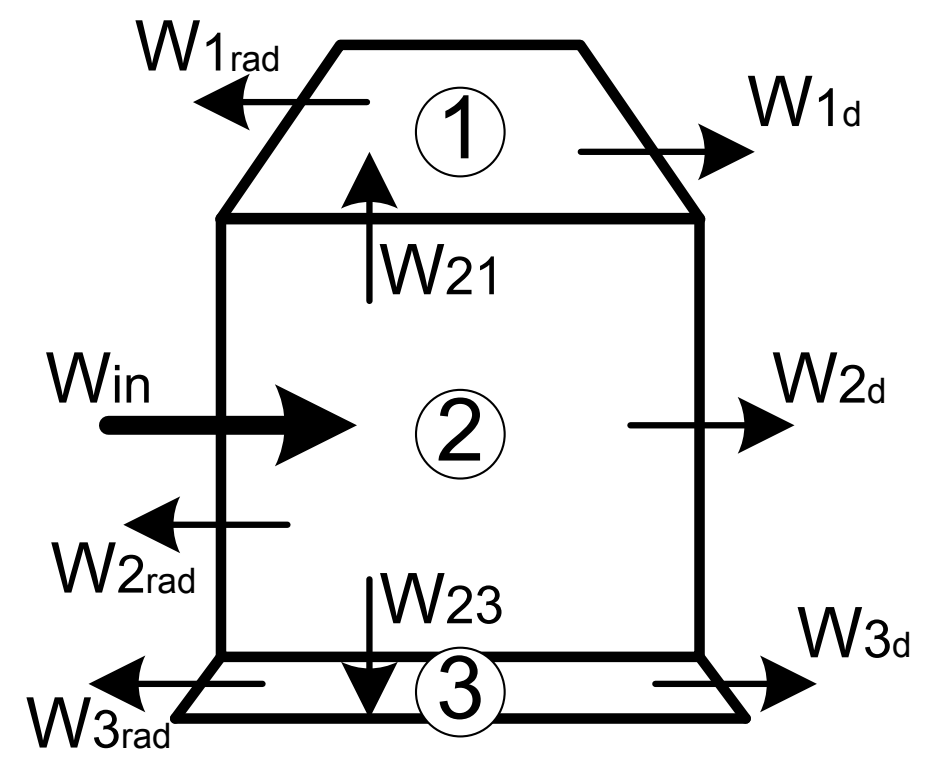

Figure 14: Power exchange between the subsystems, considered for the SEA analysis of the SYLDA.

$$
\begin{aligned}
& \frac{E_{1}}{E_{2}}=\frac{\mathbf{B}_{1,1}}{\mathbf{B}_{2,1}} \\
& \frac{E_{3}}{E_{2}}=\frac{\mathbf{B}_{3,1}}{\mathbf{B}_{2,1}}
\end{aligned}
$$

with $\mathbf{B}=\mathbf{A}^{-1}\left\{\begin{array}{c}0 \\ 1 \\ 0\end{array}\right\}$. The CLF are calculated as described above, with the assumption that the shells are behaving as flat panels above their maximum ring frequency value. It is noted that the analysis is conducted for a flexural/flexural wave transmission between the structures, as this produces the vast majority of the out of plane vibration of the shells. Once $\eta_{21}$ and $\eta_{23}$ are known, $\eta_{12}$ and $\eta_{32}$ can be calculated using the reciprocity relationship:

$$
\frac{\eta_{i j}}{\eta_{j i}}=\frac{n_{j}}{n_{i}}
$$

The modal density of the cylindrical shell is calculated by supposing simply supported boundary conditions along the edges of the shell and by subsequently interpolating on the computed frequency-wavenumber database for computing the number of resonances per frequency band. It is reminded that the assumption of any type of boundary conditions has very little influence on the modal density above the low frequency range. The same approach was adopted for the conical shells by considering their mean radius $R=\left(R_{\max }+R_{\min }\right) / 2$.

The radiation efficiency for the cylindrical shell is calculated by separating the modes into acoustically fast and acoustically slow as described in [23]. For the conical shells the same approach is used, with the assumption that their 


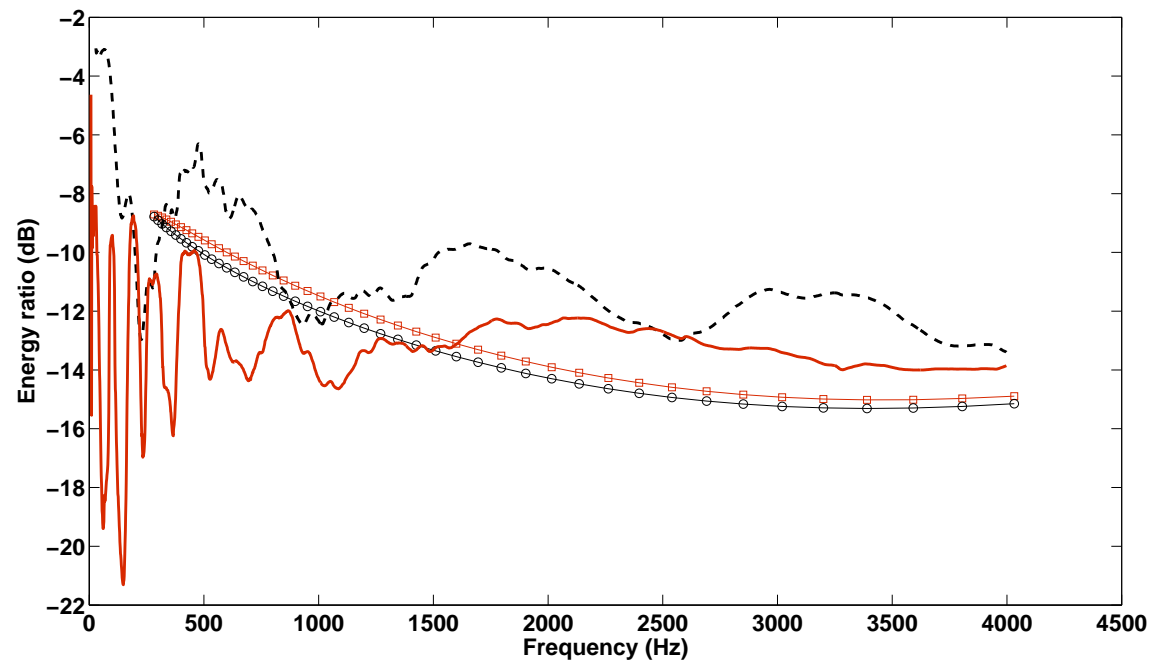

Figure 15: Energy ratio predictions $E_{1} / E_{2}$ :experimental measurements (1/6 octave averaged) (-), WFEM/SEA approach ( $\square$ ). Energy ratio predictions $E_{3} / E_{2}$ : experimental measurements (1/6 octave averaged) (--), WFEM/SEA approach (o)

radius $R$ is equal to their mean radius.

\subsubsection{Comparison between experimental and numerical results}

The experimentally measured energy ratios are post-processed as in sec.3.1. The predictions of the presented approach are then compared to the experimental values in Fig.15.

As expected, the experimental results present large fluctuations in the low frequency range where the coupling between the systems varies intensely with the presence of global system modes (modes having comparable modal displacements throughout more than one subsystems). An SEA approach may therefore not accurately model the systems response under these circumstances. For higher frequencies however, regarding the upper cone's response the WFEM/SEA approach is in very good correlation with the experimental results with the discrepancies varying from 0.9 to $1.6 \mathrm{~dB}$ above $1500 \mathrm{~Hz}$. The numerical approach seems to underestimate the energy ratio probably due to parametric uncertainties that are not taken into account and possibly due to the assumptions adopted in the calculation of the radiation efficiency and the modal density of the conical shells. With regard to the prediction of the lower cone's response, discrepancies between 3 and $5 \mathrm{~dB}$ are observed above $1500 \mathrm{~Hz}$. The experimental response of the upper shell presents intense fluctuations throughout the frequency range of measurements. This fact suggests that the shell can not be considered as weakly coupled to the cylindrical structure due to the existence of global modes between the cylindrical and the lower conical shells, throughout the considered frequency range. The 'weak coupling' assumption is central to the robustness of the SEA approach. A deterministic approach that takes into account for the individual modal coupling between the subsystems such as the FEM or an SEA-like method (see [24]) would therefore be more suitable for modelling the response of the lower conical shell. 


\section{Conclusions}

The response of an aerospace shell configuration comprising conical and cylindrical layered divisions was hereby numerically calculated. For this reason the WFEM results were implemented within a FE approach in order to model the shell's response in the low and medium frequency range, while for higher frequencies an SEA approach was adopted with the CLF between the substructures being derived by the WFEM results. To summarize the most important points of the presented work: 1) A dynamic stiffness ESL approach coupled to an FE modelling was successfully used in order to predict the dynamic response of the structure. The conical shells were considered to be divided into locally cylindrical parts in order for the technique to be applied. 2) The approach can be described as satisfactorily accurate while being computationally efficient compared to 3D FE modelling and simple implement. 3) In the high frequency range the WFEM was used in order to revisit the calculation of structural CLF between the shells and the system was modelled within an SEA approach. 4) The predictions for the upper conical shell seem to be in very good agreement with the experimental results in the high frequency range due to the fact that the shell exhibits a local modal behaviour and can properly be modelled within an SEA approach. 5) However the coupling between the cylindrical and the lower conical subsystems seems to remain strong throughout the considered frequency range. The robustness of an SEA analysis in this range is therefore questioned as the weak coupling assumption is not satisfied.

\section{Acknowledgements}

The authors would like to gratefully acknowledge the ITN Marie Curie project GA-214909 'MID-FREQUENCY: CAE Methodologies for Mid-Frequency Analysis in Vibration and Acoustics' for the financial support.

\section{References}

[1] A. Kalnins, Free vibration of rotationally symmetric shells, Journal of the Acoustical Society of America 36 (1964) $1355-65$.

[2] W. C. L. Hu, J. P. Raney, Experimental and analytical study of vibrations of joined shells, Am.Inst.Aeronautics Astronautics J. 5 (1967) 976-80.

[3] T. Irie, G. Yamada, Y. Muramoto, Free vibration of joined conical-cylindrical shells, Journal of Sound and Vibration 95 (1984) $31-9$.

[4] B. P. Patel, M. Ganapathi, S. Kamat, Free vibration characteristics of laminated composite joined conical-cylindrical shells, Journal of Sound and Vibration 237 (2000) 920-30.

[5] A. A. El Damatty, M. S. Saafan, A. M. I. Sweedan, Dynamic characteristics of combined conical-cylindrical shells, Thin-Walled Structures 43 (2005) 1380-97.

[6] E. Efraim, M. Eisenberger, Exact vibration frequencies of segmented axisymmetric shells, Thin-Walled Structures 44 (2006) 281-9.

[7] M. Caresta, N. J. Kessissoglou, Free vibrational characteristics of isotropic coupled cylindrical-conical shells, Journal of Sound and Vibration 329 (2010) 733-51.

[8] J. Kang, Three-dimensional vibration analysis of joined thick conical - cylindrical shells of revolution with variable thickness, Journal of Sound and Vibration 331 (2012) 4187-98.

[9] J. L. Rose, R. W. Mortimer, A. Blum, Elastic-wave propagation in a joined cylindrical-conical-cylindrical shell, Experimental Mechanics 13 (1973) 150-6. 
[10] R. S. Langley, Elastic wave transmission coefficients and coupling loss factors for structural junctions between curved panels, Journal of Sound and Vibration 169 (1994) 297-317.

[11] R. S. Langley, K. H. Heron, Elastic wave transmission through plate/beam junctions, Journal of Sound and Vibration 143 (1990) $241-53$.

[12] D. J. Mead, A general theory of harmonic wave propagation in linear periodic systems with multiple coupling, Journal of Sound and Vibration 27 (1973) 235-60.

[13] E. Manconi, B. R. Mace, Wave characterization of cylindrical and curved panels using a finite element method, Journal of the Acoustical Society of America 125 (2009) 154-63.

[14] D. Chronopoulos, B. Troclet, O. Bareille, M. Ichchou, Modeling the response of composite panels by a dynamic stiffness approach, Composite Structures 96 (2013) $111-20$.

[15] D. Chronopoulos, M. Ichchou, B. Troclet, O. Bareille, Efficient prediction of the response of layered shells by a dynamic stiffness approach, Composite Structures 97 (2013) $401-4$.

[16] . EADS Astrium, Functional File for SYLDA5 EGA PA2 configuration, Internal Document Ref: TE911 No:149804 (2003).

[17] J. Mencik, M. N. Ichchou, Multi-mode propagation and diffusion in structures through finite elements, European Journal of Mechanics, A/Solids 24 (2005) 877-98.

[18] J. Renno, B. Mace, Calculation of reflection and transmission coefficients of joints using a hybrid finite element/wave and finite element approach, Journal of Sound and Vibration (2012). Article in Press.

[19] W. X. Zhong, F. W. Williams, On the direct solution of wave propagation for repetitive structures, Journal of Sound and Vibration 181 (1995) $485-501$.

[20] M. Ichchou, S. Akrout, J. Mencik, Guided waves group and energy velocities via finite elements, Journal of Sound and Vibration 305 (2007) 931-44.

[21] J. Renno, E. Manconi, B. Mace, Calculation of coupling loss factors using a hybrid finite element / wave and finite element approach, in: Proceedings of the NOVEM2012, Sorrento, Italy.

[22] L. Cremer, M. Heckl, B. A. T. Petersson, Structure-borne sound: Structural vibrations and sound radiation at audio frequencies 3 (2005) $54-5$.

[23] E. Szechenyi, Modal densities and radiation efficiencies of unstiffened cylinders using statistical methods, Journal of Sound and Vibration 19 (1971) 65-81.

[24] B. R. Mace, Statistical energy analysis: Coupling loss factors, indirect coupling and system modes, Journal of Sound and Vibration 279 (2005) 141-70.

[25] M. S. Qatu, Vibration of laminated shells and plates (2004).

\section{Appendix A. Comparison of the 3D FEM predictions to experimental measurements}

The SYLDA structure is hereby numerically modelled by a full 3D FE mesh. The mesh comprises 6048 shell elements for the facesheets, coupled to 3024 solid elements which are used to model the core of the sandwich material. Perfect continuous connections are assumed at the interfaces of the conical and the cylindrical substructures. A modal numerical analysis of the composite shell structure is firstly conducted.

Using the experimentally obtained complex FRF of 792 measured points on the SYLDA, the vibrational motion of the structure was illustrated for each frequency and phase using polar coordinates in MATLAB. By investigating the obtained FRF, the experimentally measured natural frequencies in the low and mid-frequency ranges were detected and compared to the numerically predicted ones. Some representative natural frequencies along with their mode shapes are compared in Figs.A.16-A.18. 


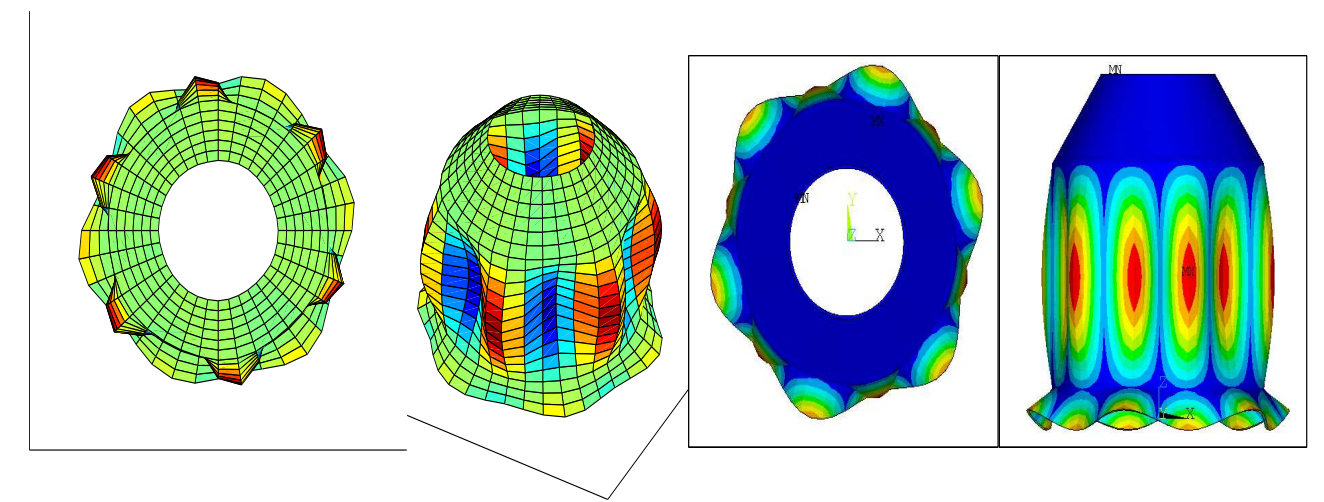

Figure A.16: The global circumferential mode of order 6: Measured (left) at $387.5 \mathrm{~Hz}$, FE prediction (right) at $386.7 \mathrm{~Hz}$

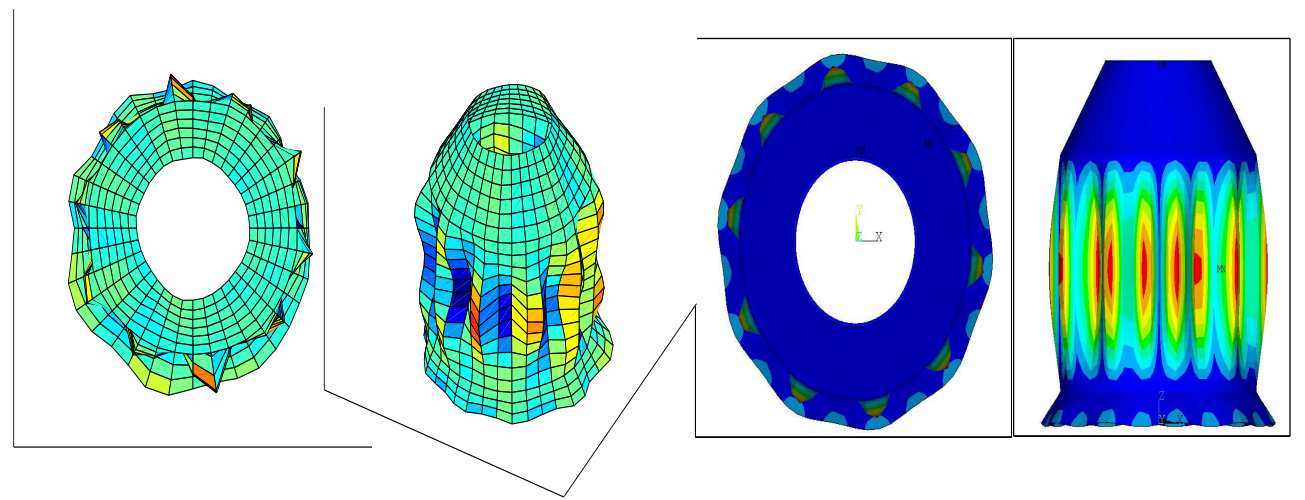

Figure A.17: The global circumferential mode of order 10: Measured (left) at $741.5 \mathrm{~Hz}$, FE prediction (right) at $732.2 \mathrm{~Hz}$ 


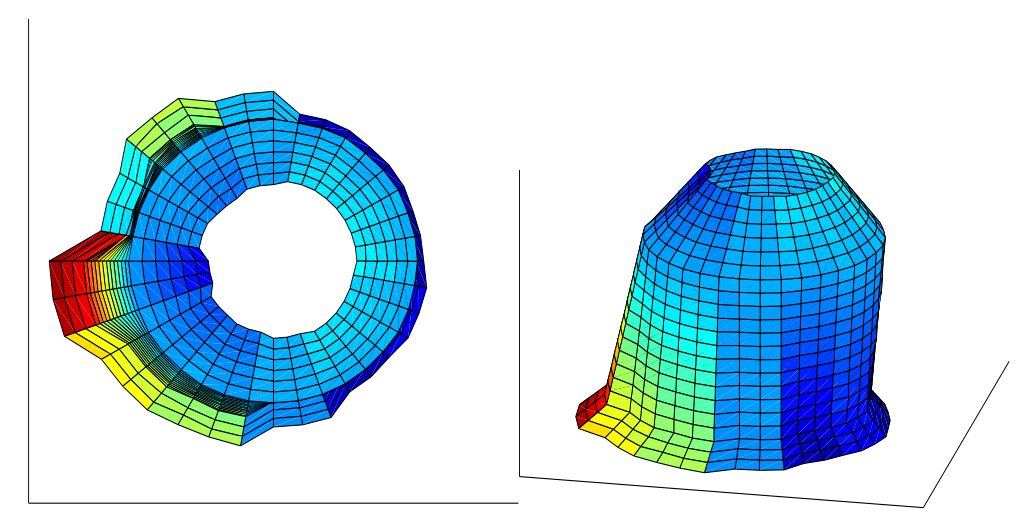

Figure A.18: The numerically non predicted fundamental mode of order 1: Measured at $8.8 \mathrm{~Hz}$

In Figs.A.16-A.17 the circumferential global modes of order 6 and 10 are exhibited as an example. It can be seen that an excellent agreement exists between the predicted and the measured natural frequencies, with the relative difference being less than 1.5\%. Regarding the mode shapes of the natural frequencies, an excellent correlation is also observed between the predicted and the measured deformations. It can also be observed that while the cylindrical part of the structure along with the lower conical part seem to have a common global motion for all modal displacements, the upper conical part does not participate in this global structural motion. Thus the upper part presents a local subsystem behaviour already in the low frequency range.

In Fig.(A.18) the circumferential mode resulted by the passage of a single circumferential flexural wave (therefore of order 1) is presented. The particularly responsive fundamental mode is experimentally observed however it is not numerically predicted by the FE model. This difference in the fundamental frequency prediction can be crucial for low frequency excitations. The fundamental frequency prediction by analytical models usually raises with the thickness ratio of the shells and is discussed in [25].

Following the modal analysis, a harmonic analysis was numerically conducted. In cylindrical coordinates, the excitation force is fixed at $(\theta, z)=\left(0^{\circ}, 494 \mathrm{~mm}\right)$ position of the cylindrical part. The resulting velocity FRF at arbitrary points of the three substructures are presented in Figs.A.19-A.21.

The measured and the FE predicted velocity FRF at an arbitrary point of the cylindrical section is presented in Fig.A.19. An excellent agreement between the experimental results and the numerical predictions is observed within the low frequency range. With the exception of the fundamental frequency (as discussed above), the entirety of the global modal behaviour of the structure is very well predicted throughout the presented frequency range. Experimental values were used for the structural damping loss factor. The modal peaks corresponding to peripheral modes of the cylinder tend to be the most responsive ones.

The measured and the FE predicted velocity FRF at arbitrary points of the lower conical section are presented in Fig.A.20 and for the upper conical part in Fig.A.21. For the lower conical part a fairly good agreement of the response is observed up until $500 \mathrm{~Hz}$ with the predicted resonances and anti-resonances being well correlated with the measured 


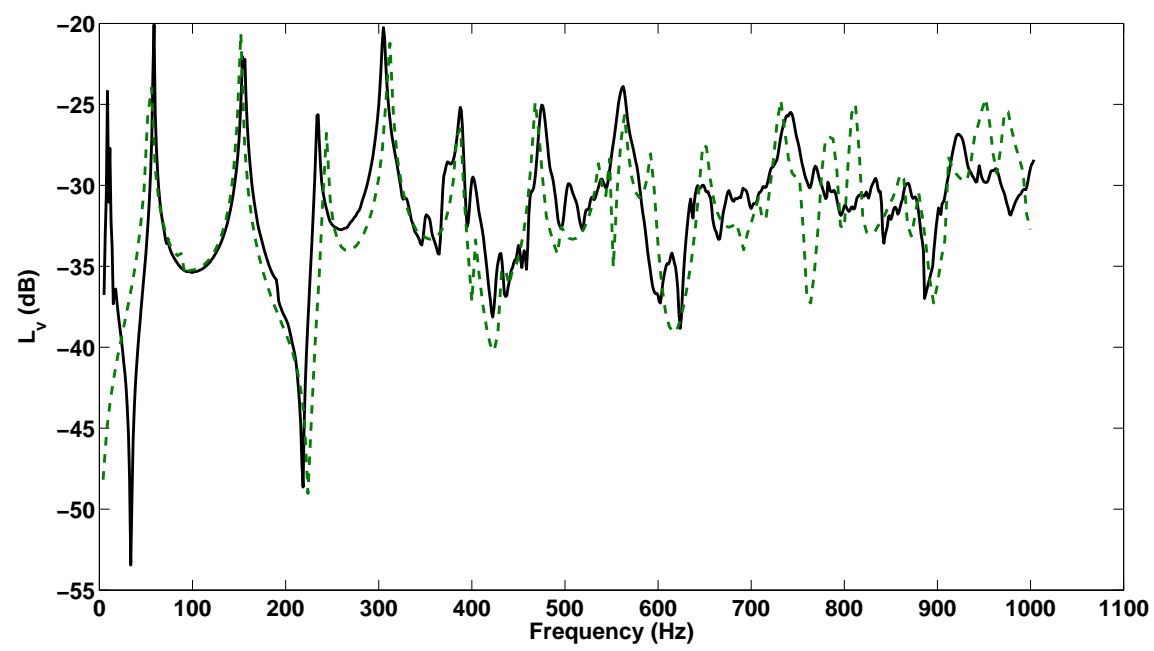

Figure A.19: Velocity FRF level comparison at $\left(250^{\circ}, 263 \mathrm{~mm}\right)$ of the cylindrical part: Experimental results (-), FEM results $(--)$

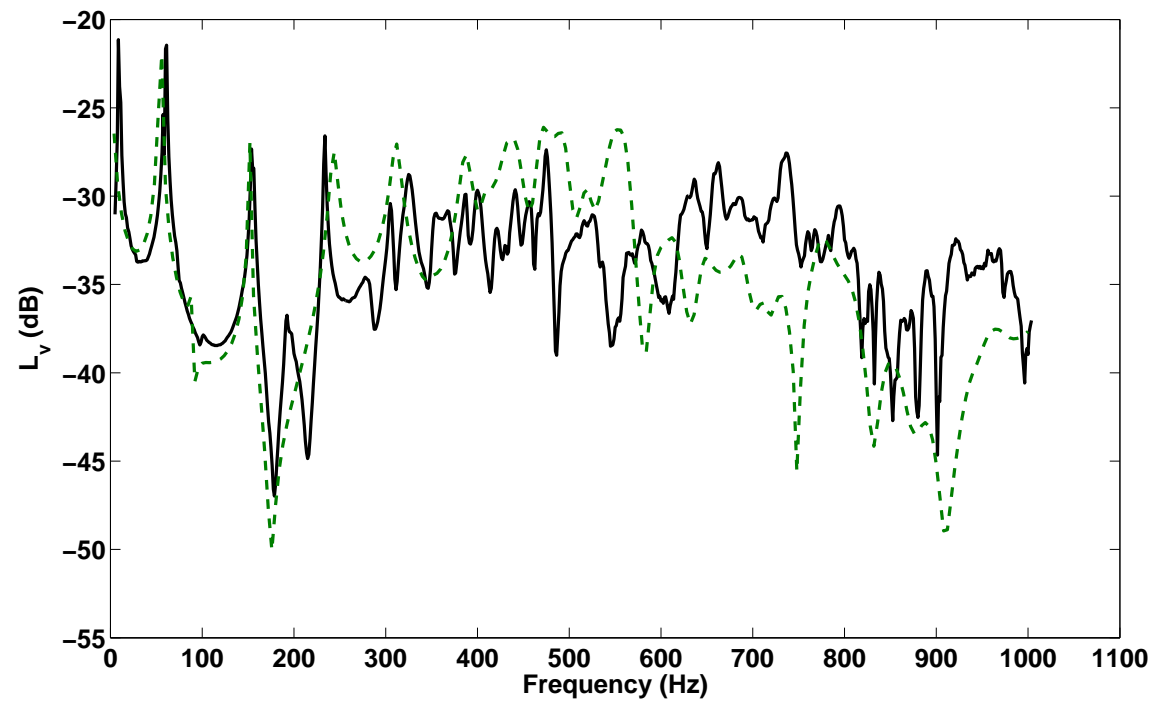

Figure A.20: Velocity FRF level comparison at $\left(180^{\circ}, 50 \mathrm{~mm}\right)$ of the lower conical part: Experimental results (-), FEM results (- -) 


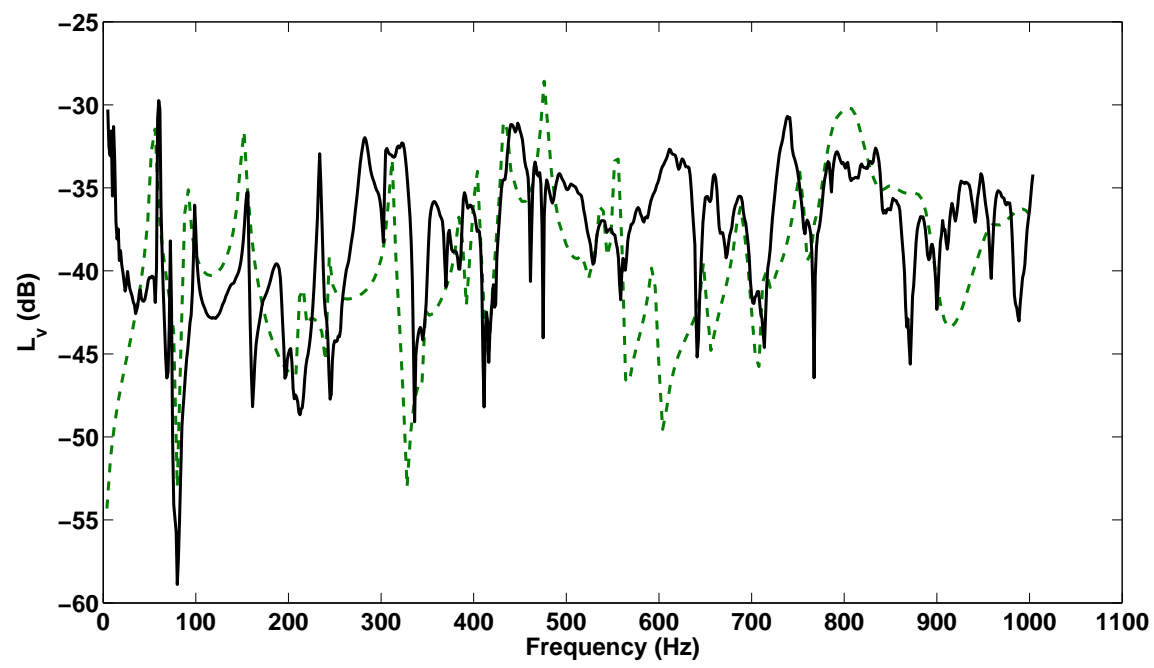

Figure A.21: Velocity FRF level comparison at $\left(250^{\circ}, 219 \mathrm{~mm}\right)$ of the upper conical part: Experimental results (-), FEM results (- -)

ones. For higher frequencies however the velocities of both the presented points seem to be underestimated by the FEM, implying a coupling greater than the one predicted. Observing the high number of peaks of the experimental curve in this frequency range, this can be attributed to the effect of local modes of other substructures on the response of the lower cone, including indirect coupling with the upper cone modes. With regard to the upper conical structure, the response also seems to be generally well predicted by the FE model with the exception of a frequency range around $600 \mathrm{~Hz}$ where the response seems to have been underestimated. Comparing the FRF of the cylindrical part to the ones obtained on the conical parts, it is seen that the later contain many more peaks, despite the fact that conical parts are substantially smaller than the cylindrical one. This stresses the impact that the modes of the cylindrical part have on the response of the conical shells and indicates the complicated nature of waves travelling in a conical structure.

In order to get a more global idea of how the predicted response compares to measurements, the total energy of each subsystem was calculated using the predicted and measured force normalized velocities. The results are presented in Figs.A.22,A.23,A.24.

Regarding the cylindrical part is it observed that the FE predicted subsystem energy is very much in accordance with the measured one throughout the considered frequency band. As aforementioned, the FEM seems to be underestimating the effect of local modes of the conical parts on the energy of the cylindrical part, especially for frequencies between the resonances where this effect becomes evident. The energy of the upper conical part (along with the impact of the upper cone local modes) seems to be overestimated up to $150 \mathrm{~Hz}$ and afterwards underestimated until $400 \mathrm{~Hz}$. It is noted as an example that despite the fact that the upper cone local mode of order 3 is well predicted by the FE modal analysis at $217 \mathrm{~Hz}$, in Fig.A.23 this mode is not observable. Better correlation is observed for higher frequencies. With regard to the upper conical part, it can be seen that it follows a global modal behaviour, influenced by the modes of the cylindrical part up until $300 \mathrm{~Hz}$. While beyond this frequency the cylindrical modes are apparent, a number of local modes can also be observed resulting in a local-global behaviour. At higher frequencies the cou- 


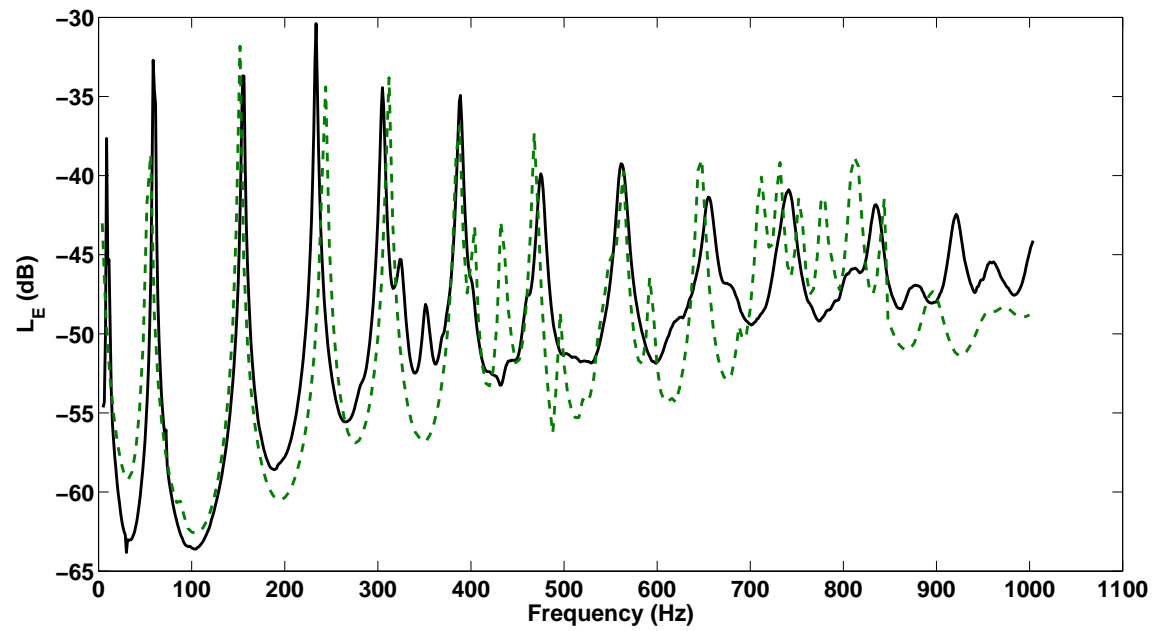

Figure A.22: Total energy level of the cylindrical subsystem: Experimental results (-), FEM results (- -)

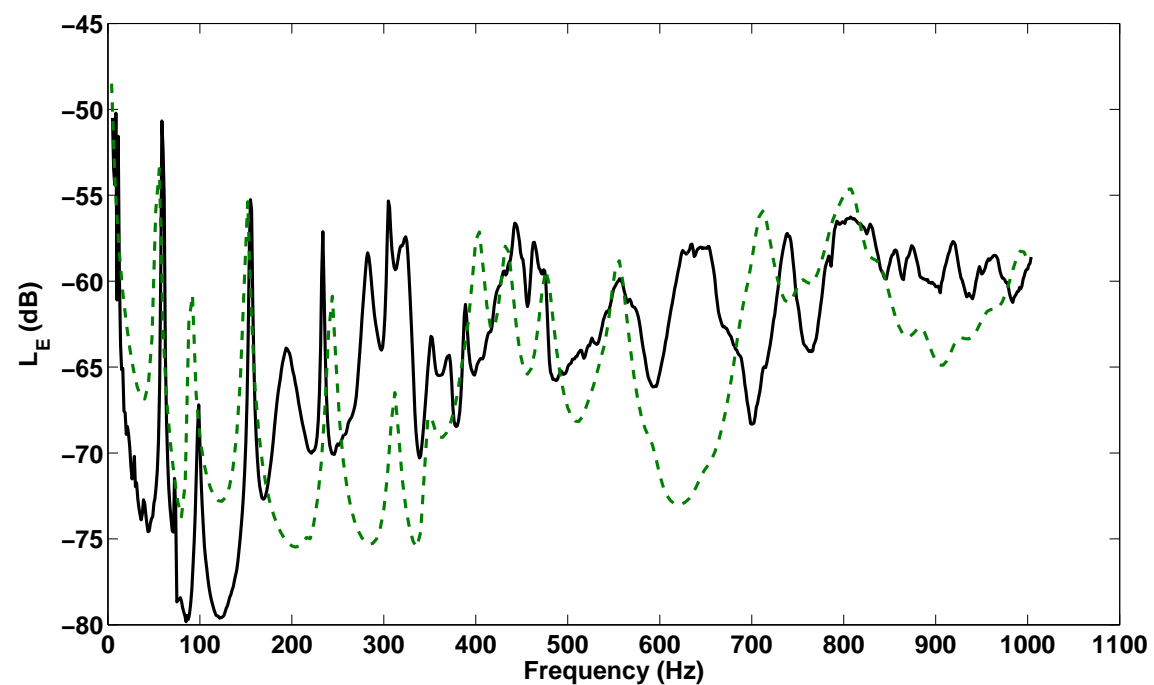

Figure A.23: Total energy level of the upper conical subsystem: Experimental results (-), FEM results (- -) 


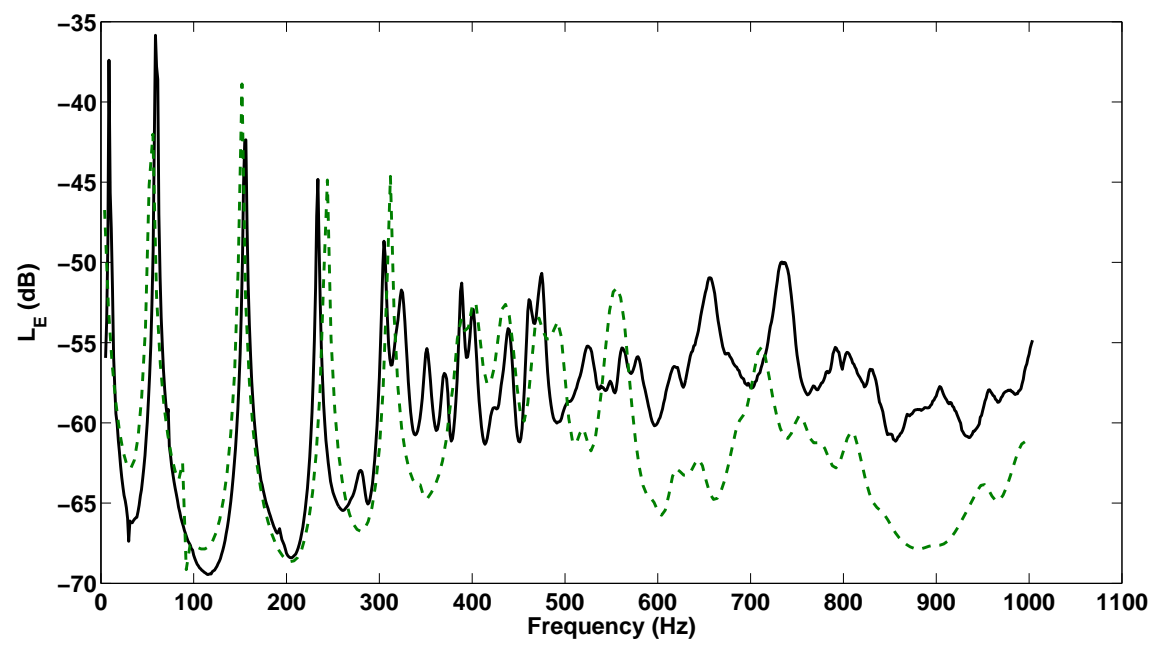

Figure A.24: Total energy level of the lower conical subsystem: Experimental results (-), FEM results (--)

pling of the lower cone to the rest of the structure is underestimated by the FE predictions, probably due to geometric uncertainties at the cylindrical conical interfaces as aforementioned. 